Part of Journal of Research of the National Bureau of Standards, Volume 19, December 1937

\title{
ARC AND SPARK SPECTRA OF YTTERBIUM
}

\author{
By William F. Meggers and Bourdon F. Scribner
}

\section{ABSTRACT}

A new description of conventional arc and spark spectra of ytterbium (neoytterbium, aldeberanium) has been completed in the wave-length range 2000 to $11000 \mathrm{~A}$. Wave-length measurements and intensity estimates are presented for 1,668 lines, 400 of which characterize neutral $\mathrm{Yb}$ atoms ( $\mathrm{Yb}$ I spectrum), about 1,250 are due to singly ionized atoms ( $\mathrm{Yb}$ II spectrum), and possibly a dozen belong to doubly ionized atoms ( $\mathrm{Yb}$ III spectrum). Some spectral regularities are given for neutral and for singly ionized atoms. The ground states of $\mathrm{Yb}$, $\mathrm{Yb}^{+}$, and $\mathrm{Yb}^{++}$atoms appear to be associated with the electron configurations, $4 f^{14} 6 s^{2}, 4 f^{14} 6 s$, and $4 f^{14}$, respectively.

\section{CONTENTS}

I. Introduction

II. Experiments.

III. Results _. .

IV. References

\section{INTRODUCTION}

In 1878, Marignac [1] ${ }^{1}$ discovered in gadolonite a new earth which he named ytterbium ( $\mathrm{Yb}$ ). Nearly 30 years later Urbain [2] succeeded in splitting ytterbium into two components, which he named neoytterbium $(\mathrm{Ny})$ and lutecium $(\mathrm{Lu})$. The same separation was made independently (and probably somewhat earlier) by Auer [3], who proposed the names aldeberanium (Ad) and cassiopeium (Cp). The common usage now is to represent the elements having atomic numbers 70 and 71 by the symbols $\mathrm{Yb}$ and $\mathrm{Lu}$, respectively, except in Austria and Germany where Ad and $\mathrm{Cp}$ are more generally used.

Owing to the difficulty of separating rare earths from one another, and to the complex nature of their spectra, descriptions of the latter have long been in a most unsatisfactory state. In our recent paper [4] on the arc and spark spectra of lutecium, we remarked that all available samples of lutecium were contaminated by ytterbium and thulium so that the spectra of the former could not be unambiguously determined without simultaneous comparison with ytterbium and thulium spectra. Thus our attempt to produce a complete and trustworthy description of lutecium spectra naturally led to the production of similar data for thulium and ytterbium. Improved descriptions of these spectra are essential not only for purposes of spectroscopic identification and analysis, but also for the term analyses and interpretation or spectral structures characteristic of different

\footnotetext{
1 Numbers in brackets refer to literature citations at the end of this paper.
} 
atoms and ions. In the present paper we make public our latest description of the conventional arc and spark spectra of ytterbium, including some preliminary details about spectral structures.

The only spectroscopic tables published for ytterbium since the discovery of lutecium are the following [5]. Auer [3] first gave a list of 200 spark spectrum lines (2621.24 to 5897.56 A), but most of the wave lengths were borrowed from a table which Exner and Haschek had given for old ytterbium containing unrecognized lutecium. In 1910, Eder and Valenta [6] published 58 lines (5474.24 to $5788.87 \mathrm{~A}$ ) observed in the arc spectrum and in 1911, reproduced spectrograms and gave tables of wave lengths [7] for the arc spectrum (169 lines 2615.48 to $6799.87 \mathrm{~A}$ ) and for the spark spectrum (195 lines 2615.48 to $6799.87 \mathrm{~A}$ ). The most complete tables were published, in 1911, by Exner and Haschek, who list 905 lines (2320.95 to $6799.91 \mathrm{~A}$ ) observed in the arc spectrum [8] and 795 lines (2224.58 to $6489.31 \mathrm{~A}$ ) in the spark spectrum [9]. These tables contain a considerable number of lutecium and thulium lines, and many ytterbium lines are common to both, without positive indication as to whether they belong to neutral or to ionized atoms. In 1914, Blumenfeld and Urbain [10] measured the wave lengths of 499 lines (2309.6 to $3499.4 \mathrm{~A}$ ) most of which were believed to represent ytterbium, although many were recognized as possible impurities. The latest extensive description of $\mathrm{Yb}$ spectra consists of 422 lines (2271.51 to 7699.49 A) observed in the arc spectrum by Eder [11], neglecting more than 200 lines ascribed to impurities, principally Tm. The salts available for these early investigations of rare-earth spectra were so impure that great difficulty was experienced in sorting the lines and frequently the same lines were ascribed to two different elements. For example, [12] $2615.41 \mathrm{Yb}, \mathrm{Lu} ; 2693.33 \mathrm{Yb}$, $\mathrm{Lu} ; 3283.40 \mathrm{Yb}$, Tm; $3362.61 \mathrm{Yb}$, Tm; $3425.10 \mathrm{Yb}$, Tm; $3441.51 \mathrm{Yb}$, Tm; 3472.48 Yb, Lu; 3887.34 Yb, Er; 5074.32, De, Tm, Yb; 5307.11 $\mathrm{Yb}$, Tm. Except for the temperature classification of 399 lines (2935.09 to 6799.58 A) by King [13], no further investigations of $\mathrm{Yb}$ spectra have been reported in the past 22 years.

\section{EXPERIMENTS}

Our first measurements of $\mathrm{Yb}$ spectra were made in 1929 when the investigation of $\mathrm{Lu}$ spectra was begun [4]. Samples of $\mathrm{Lu}$ and $\mathrm{Yb}$ oxides prepared by Auer, and obtained from Eder in 1919 when he discontinued his work in spectroscopy, were available. Altbough the chemical separation was far from complete, it was possible to make a fairly satisfactory assignment of lines to $\mathrm{Lu}$ and $\mathrm{Yb}$ by simultaneous comparison of arc and spark spectra. However, both salts contained some Tm as impurity which could not be completely identified without comparable data for Tm spectra. The first sample of Tm salt available for this purpose was generously supplied in 1933, by Professor B. S. Hopkins, University of Illinois, who also supplied a sample of $\mathrm{Lu}$ oxide and three samples of $\mathrm{Yb}$ oxide, one of which contained more Lu than Tm, another more Tm than Lu, and the third was entirely free from both $\mathrm{Lu}$ and $\mathrm{Tm}$, but contained considerable lanthanum. This ytterbium material was purified by the electrolytic reduction method devised by L. F. Yntema [14]. Alkali (Li, Na, K) and alkaline-earth $(\mathrm{Mg}, \mathrm{Ca}, \mathrm{Sr}, \mathrm{Ba})$ impurities are usually present in rare-earth salts, but these have extremely simple spectra, which are 
easily identified. Miscellaneous impurities were detected by comparing our final wave-length list with Kayser's Hauptlinien [12] and with a description of lanthanum spectra [15].

The procedures employed to insure the correct assignment of spectral lines to their emitter, both as regards chemical element and stage of ionization, were the same as for the study of Lu spectra [4], and the details regarding light sources, spectrographs, photographic plates, etc., given there need not be repeated here.

\section{RESULTS}

The measured wave lengths and estimated relative intensities of $1,668 \mathrm{Yb}$ lines observed in arc and spark spectra are presented in table 1. Each line in this table was observed on two or more spectrograms except a few with questioned intensities which were seen only on the strongest exposures. The brightest $\mathrm{Yb}$ lines were measured on eight or nine spectrograms, and many of them were measured a number of times on $\mathrm{Lu}$ and Tm spectrograms, where they appeared as impurities. The concordance of values from different spectrograms and the coincidence of wave lengths with impurity lines indicates that the final results for most lines are correct within $0.01 \mathrm{~A}$, but errors of $0.02 \mathrm{~A}$ or more may exist among faint lines. Hazy lines which occur so frequently in spark spectra are not only subject to larger accidental errors but may show some systematic error, since they are usually unsymmetrical. Otherwise, Yb lines appear to be sharp and free from hyperfine structure. No information on the isotopic constitution of $\mathrm{Yb}$ is available, but since hyperfine structures have been detected only for lines of rare earths with odd atomic number, $\mathrm{Yb}(\mathrm{Z}=70)$ may be assumed to be immune.

In 1915 Eder [11] published 177 lines, which he suspected belonged to a new element, denebium (De). It now appears that at least 110 of these lines are due to ytterbium, and many of the remainder are due to thulium.

TABLE 1.-Arc and spark spectra of ytterbium $(\mathrm{Z}=70)$

$d=$ Double.

$e=$ Enhanced at electrode. $h=$ Hazy.

$H=$ Very hazy.

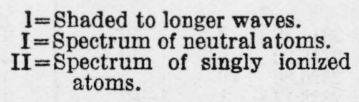

$1=$ Shaded to longer waves. $\mathbf{I}=$ Spectrum of neutral atoms. II = Spectrum of singly ionized atoms.

\begin{tabular}{|c|c|c|c|c|c|c|c|c|c|c|c|}
\hline \multirow{2}{*}{$\lambda_{\text {sir }} \mathrm{A}$} & \multicolumn{2}{|c|}{$\begin{array}{l}\text { Intensity and } \\
\text { character }\end{array}$} & \multirow{2}{*}{$\begin{array}{l}\text { Spec- } \\
\text { trum }\end{array}$} & \multirow{2}{*}{$\lambda$ air $A$} & \multicolumn{2}{|c|}{$\begin{array}{l}\text { Intensity and } \\
\text { character }\end{array}$} & \multirow{2}{*}{$\begin{array}{l}\text { Spec- } \\
\text { trum }\end{array}$} & \multirow{2}{*}{$\lambda$ ir $A$} & \multicolumn{2}{|c|}{$\begin{array}{l}\text { Intensity and } \\
\text { character }\end{array}$} & \multirow{2}{*}{$\begin{array}{l}\text { Spec- } \\
\text { trum }\end{array}$} \\
\hline & Arc & Spark & & & Arc & Spark & & & Aro & Spark & \\
\hline $\begin{array}{l}2073.70 \\
2078.12 \\
2083.65 \\
2087.37 \\
2088.05\end{array}$ & \begin{tabular}{|c|} 
\\
\end{tabular} & $\begin{array}{c}8 h \\
10 h \\
2 \\
3 h \\
15 h\end{array}$ & $\begin{array}{l}\text { II } \\
\text { II } \\
\text { II } \\
\text { II } \\
\text { II }\end{array}$ & $\begin{array}{l}2102.72 \\
2106.46 \\
2108.20 \\
2109.60 \\
2110.26\end{array}$ & \begin{tabular}{|c|}
20 \\
\end{tabular} & $\begin{array}{r}200 \\
2 \\
5 \\
100 h \\
5 h\end{array}$ & $\begin{array}{l}\text { II } \\
\text { II } \\
\text { II } \\
\text { II } \\
\text { II }\end{array}$ & $\begin{array}{l}2127.54 \\
2128.82 \\
2129.64 \\
2130.45 \\
2131.37\end{array}$ & 10 & $\begin{array}{c}2 h \\
2 \\
4 h \\
2 h \\
20\end{array}$ & $\begin{array}{l}\text { II } \\
\text { II } \\
\text { II } \\
\text { II } \\
\text { II }\end{array}$ \\
\hline $\begin{array}{l}2091.28 \\
2092.31 \\
2093.18 \\
2094.82 \\
2095.38\end{array}$ & \begin{tabular}{|c|} 
\\
-2
\end{tabular} & $\begin{array}{r}20 h \\
30 h \\
6 h \\
10 h \\
200 h\end{array}$ & $\begin{array}{l}\text { II } \\
\text { II } \\
\text { II } \\
\text { II } \\
\text { III? }\end{array}$ & $\begin{array}{l}2110.60 \\
2115.46 \\
2115.84 \\
2116.65 \\
2117.83\end{array}$ & - 50 & $\begin{array}{c}3 \\
4 h \\
2 \\
250 \\
4 h\end{array}$ & $\begin{array}{l}\text { II } \\
\text { II } \\
\text { II } \\
\text { II } \\
\text { II }\end{array}$ & $\begin{array}{l}2131.71 \\
2133.17 \\
2133.34 \\
2133.66 \\
2134.98\end{array}$ & $\mid \begin{array}{l} \\
\end{array}$ & $\begin{array}{l}2 h \\
3 \\
3 \\
4 h \\
3 h\end{array}$ & $\begin{array}{l}\text { II } \\
\text { II } \\
\text { II } \\
\text { II } \\
\text { II }\end{array}$ \\
\hline $\begin{array}{l}2096.84 \\
2097.41 \\
2098.16 \\
2098.40 \\
2102.10\end{array}$ & 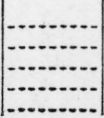 & $\begin{array}{c}30 h \\
2 \\
3 \\
50 h \\
3 h\end{array}$ & $\begin{array}{l}\text { II } \\
\text { II } \\
\text { II } \\
\text { II } \\
\text { II }\end{array}$ & $\begin{array}{l}2119.25 \\
2121.54 \\
2122.81 \\
2123.30 \\
2126.72\end{array}$ & (40 & $\begin{array}{c}30 h \\
15 \\
3 \\
15 \\
200\end{array}$ & $\begin{array}{l}\text { II } \\
\text { II } \\
\text { II } \\
\text { II } \\
\text { II }\end{array}$ & $\begin{array}{l}2135.22 \\
2136.33 \\
2137.52 \\
2137.71 \\
2138.32\end{array}$ & $\left|\begin{array}{|c|} \\
\end{array}\right|$ & $\begin{array}{l}20 h \\
2 \\
9 \\
15 \\
10\end{array}$ & $\begin{array}{l}\text { II } \\
\text { II } \\
\text { II } \\
\text { II } \\
\text { II }\end{array}$ \\
\hline
\end{tabular}


TABLE 1.-Arc and spark spectra of ytterbium $(\mathrm{Z}=70)$-Continued

\begin{tabular}{|c|c|c|c|c|c|c|c|c|c|c|c|}
\hline \multirow{2}{*}{$\lambda_{\text {sir }} \mathrm{A}$} & \multicolumn{2}{|c|}{$\begin{array}{l}\text { Intensity and } \\
\text { character }\end{array}$} & \multirow{2}{*}{$\begin{array}{l}\text { Spec- } \\
\text { trum }\end{array}$} & \multirow{2}{*}{$\lambda$ air $\mathbf{A}$} & \multicolumn{2}{|c|}{$\begin{array}{l}\text { Intensity and } \\
\text { character }\end{array}$} & \multirow{2}{*}{$\begin{array}{l}\text { Spec- } \\
\text { trum }\end{array}$} & \multirow{2}{*}{$\lambda_{\text {air }} \mathrm{A}$} & \multicolumn{2}{|c|}{$\begin{array}{l}\text { Intensity and } \\
\text { character }\end{array}$} & \multirow{2}{*}{$\begin{array}{l}\text { Spec- } \\
\text { trum }\end{array}$} \\
\hline & Arc & Spark & & & Arc & Spark & & & Arc & Spark & \\
\hline $\begin{array}{l}2139.25 \\
2139.96 \\
2140.41 \\
2141.00 \\
2141.67\end{array}$ & & $\begin{array}{r}2 \\
25 \\
2 \\
10 \\
5\end{array}$ & $\begin{array}{l}\text { II } \\
\text { II } \\
\text { II } \\
\text { II } \\
\text { II }\end{array}$ & $\begin{array}{l}2211.87 \\
2214.64 \\
2221.47 \\
2224.45 \\
2224.83\end{array}$ & $\begin{array}{c}1 \\
20 \\
-1\end{array}$ & $\begin{array}{l}2 \\
8 \\
2 h ? \\
40 \\
2\end{array}$ & $\begin{array}{l}\text { II } \\
\text { II } \\
\text { II } \\
\text { II } \\
\text { II }\end{array}$ & $\begin{array}{l}2373.89 \\
2377.32 \\
2380.37 \\
2382.56 \\
2385.00\end{array}$ & $\begin{array}{l}2 \\
-2 \\
-1\end{array}$ & $\begin{array}{l}8 \\
2 h \\
9 \\
2 \\
5\end{array}$ & \\
\hline $\begin{array}{l}2142.18 \\
2143.42 \\
2143.86 \\
2144.74 \\
2146.46\end{array}$ & & $\begin{array}{c}4 \\
5 ? \\
4 ? \\
30 \mathrm{Ag} ? \\
3 ?\end{array}$ & $\begin{array}{l}\text { II } \\
\text { II } \\
\text { II } \\
\text { II } \\
\text { II }\end{array}$ & $\begin{array}{l}2227.70 \\
2231.55 \\
2237.48 \\
2240.09 \\
2244.25\end{array}$ & 5 & $\begin{array}{c}3 \\
2 \\
3 \\
25 \\
8 h\end{array}$ & $\begin{array}{l}\text { II } \\
\text { II } \\
\text { II } \\
\text { II } \\
\text { II }\end{array}$ & $\begin{array}{l}2388.40 \\
2390.72 \\
2397.32 \\
2397.83 \\
2398.00\end{array}$ & $\begin{array}{c}1 \\
10 \\
10\end{array}$ & $\begin{array}{c}3 \\
30 \\
1 ? \\
3 \\
5\end{array}$ & \\
\hline $\begin{array}{l}2147.15 \\
2147.50 \\
2148.07 \\
2148.49 \\
2148.92\end{array}$ & & $\begin{array}{l}1 \\
3 h \\
8 \\
4 \\
10 \mathrm{Cu} ?\end{array}$ & $\begin{array}{l}\text { II } \\
\text { II } \\
\text { II } \\
\text { II } \\
\text { II }\end{array}$ & $\begin{array}{l}2255.46 \\
2257.01 \\
2261.12 \\
2262.25 \\
2263.16\end{array}$ & $\begin{array}{c}4 \\
2\end{array}$ & $\begin{array}{c}1 ? \\
40 \\
3 \\
30 \\
3 h\end{array}$ & $\begin{array}{l}\text { II } \\
\text { II } \\
\text { II } \\
\text { II } \\
\text { II }\end{array}$ & $\begin{array}{l}2403.39 \\
2404.07 \\
2406.07 \\
2418.39 \\
2421.36\end{array}$ & 1 & $\begin{array}{c}3 \\
4 h \\
5 \\
2 h \\
25\end{array}$ & \\
\hline $\begin{array}{l}2149.43 \\
2150.45 \\
2150.93 \\
2151.20 \\
2152.32\end{array}$ & & $\begin{array}{c}3 \\
3 \\
2 \\
2 h \\
10 h \\
10 h\end{array}$ & $\begin{array}{l}\text { II } \\
\text { II } \\
\text { II } \\
\text { II } \\
\text { II }\end{array}$ & $\begin{array}{l}2263.85 \\
2265.65 \\
2267.15 \\
2268.28 \\
2268.64\end{array}$ & $\begin{array}{c}3 \\
1 \\
-1\end{array}$ & $\begin{array}{r}2 \\
50 \\
1 \\
4 \\
3 h\end{array}$ & $\begin{array}{l}\text { II } \\
\text { II } \\
\text { II } \\
\text { II } \\
\text { II }\end{array}$ & $\begin{array}{l}2422.81 \\
2424.56 \\
2425.65 \\
2426.16 \\
2433.62\end{array}$ & \begin{tabular}{c}
2 \\
\hdashline- \\
\end{tabular} & $\begin{array}{l}4 \\
2 h \\
2 h \\
1 \\
6 h\end{array}$ & \\
\hline $\begin{array}{l}2153.44 \\
2153.67\end{array}$ & & $\begin{array}{l}1 \\
1\end{array}$ & II & $\begin{array}{l}2271.12 \\
2276.07 \\
2280\end{array}$ & $\begin{array}{l}8 ? \\
2 ?\end{array}$ & (- & I & 2439.30 & & 4 & \\
\hline 2154.16 & & $80 h$ & $\left\{\begin{array}{c}\text { II, } \\
\text { III? }\end{array}\right.$ & $\begin{array}{l}2282.99 \\
2283.38\end{array}$ & $\begin{array}{l}5 \\
8\end{array}$ & $\begin{array}{l}50 \\
10\end{array}$ & $\begin{array}{l}\text { II } \\
\text { II }\end{array}$ & $\begin{array}{l}2439.67 \\
2440.57\end{array}$ & & $\begin{array}{l}3 \\
5 h\end{array}$ & \\
\hline $\begin{array}{l}2155.18 \\
2155.51\end{array}$ & & $\begin{array}{r}7 \\
40\end{array}$ & $\begin{array}{l}\text { II } \\
\text { II }\end{array}$ & $\begin{array}{l}2283.98 \\
2285.80\end{array}$ & $3 ?$ & $8 h$ & II & $\begin{array}{l}2447.23 \\
2454.7\end{array}$ & & $\begin{array}{c}4 \\
20 H\end{array}$ & \\
\hline $\begin{array}{l}2156.51 \\
2156.79 \\
2157.87 \\
2158.79 \\
2159.29\end{array}$ & & $\begin{array}{l}3 \\
2 \\
3 h \\
4 \\
1\end{array}$ & $\begin{array}{l}\text { II } \\
\text { II } \\
\text { II } \\
\text { II } \\
\text { II }\end{array}$ & $\begin{array}{l}2285.85 \\
2288.93 \\
2291.63 \\
2292.27 \\
2292.81\end{array}$ & 1 & $\begin{array}{l}8 h \\
6 \\
3 h \\
3 h\end{array}$ & $\begin{array}{l}\text { II } \\
\text { II } \\
\text { II } \\
\text { II }\end{array}$ & $\begin{array}{l}2456.00 \\
2457.65 \\
2458.23 \\
2460.24 \\
2461.38\end{array}$ & 6 & $\begin{array}{c}5 h \\
2 \\
1 h \\
30 \mathrm{Ag} ? \\
5 h\end{array}$ & \\
\hline $\begin{array}{l}2159.88 \\
2160.27\end{array}$ & & $\begin{array}{r}3 h \\
10 h\end{array}$ & $\begin{array}{l}\text { II } \\
\text { II }\end{array}$ & 2297.88 & $\begin{array}{l}1 \\
2 \\
1\end{array}$ & $\begin{array}{l}4 \\
4\end{array}$ & $\begin{array}{l}\text { II } \\
\text { II }\end{array}$ & 2464.48 & 20 & $\begin{array}{l}5 \\
3 ?\end{array}$ & \\
\hline & 100 & $\begin{array}{r}250 \\
1\end{array}$ & $\begin{array}{l}\text { II } \\
\text { II }\end{array}$ & $\begin{array}{l}2303.30 \\
2305.33\end{array}$ & 8 & ${ }_{100}^{2 d \mathrm{Lu} ?}$ & $\begin{array}{l}\text { II } \\
\text { II }\end{array}$ & $\begin{array}{l}2466.62 \\
2471.04\end{array}$ & 2 & $\begin{array}{l}10 \\
2 ?\end{array}$ & \\
\hline 2163.87 & & 10 & II & & & & & 2481.40 & 1 & 4 & \\
\hline $\begin{array}{l}2165.19 \\
2165.53\end{array}$ & & $\begin{array}{l}8 h \\
3\end{array}$ & $\begin{array}{l}\text { II } \\
\text { II }\end{array}$ & $\begin{array}{l}2307.41 \\
2308.49 \\
2309.26\end{array}$ & 1 & $\begin{array}{r}6 h \\
2 h \\
20\end{array}$ & $\begin{array}{l}\text { II } \\
\text { II }\end{array}$ & $\begin{array}{l}2484.28 \\
2484.88\end{array}$ & 3 & $\begin{array}{l}2 \\
8\end{array}$ & \\
\hline & & 3 & II & & $2 ? \mathrm{Lu}$ & & I & 2487.03 & & 2 & \\
\hline & & $\begin{array}{l}7 \\
5\end{array}$ & $\begin{array}{l}\text { II } \\
\text { II }\end{array}$ & 2312.56 & 1 & 2 & II & $\begin{array}{l}2488.96 \\
2490.45\end{array}$ & 2 & $\begin{array}{r}3 \\
40\end{array}$ & \\
\hline \begin{tabular}{l|}
2172.13 \\
2172.58 \\
2173.34 \\
2174.28 \\
2175.38
\end{tabular} & $\begin{array}{l}2 \\
4\end{array}$ & $\begin{array}{c}3 \\
2 \\
5 \\
10 \\
3 h \mathrm{Lu} ?\end{array}$ & $\begin{array}{l}\text { II } \\
\text { II } \\
\text { II } \\
\text { II } \\
\text { II }\end{array}$ & $\begin{array}{l}2314.48 \\
2315.20 \\
2320.80 \\
2323.17 \\
2326.91\end{array}$ & $\begin{array}{r}4 \\
5 \\
20 \\
1 \\
2\end{array}$ & $\begin{array}{c}50 \\
10 \\
5 \\
2 \\
6 h\end{array}$ & \begin{tabular}{r|r} 
II \\
II \\
I \\
II \\
II
\end{tabular} & $\begin{array}{l}2491.69 \\
2493.63 \\
2495.03 \\
2495.69 \\
2498.35\end{array}$ & $\begin{array}{c}2 \\
1\end{array}$ & $\begin{array}{c}3 \\
5 \\
1 \\
10 h \\
4\end{array}$ & \\
\hline $\begin{array}{l}2175.88 \\
2177.04 \\
2177.50 \\
2178.80 \\
2180.24\end{array}$ & & $\begin{array}{l}1 \\
1 \\
5 \\
8 h \\
7 h\end{array}$ & $\begin{array}{l}\text { II } \\
\text { II } \\
\text { II } \\
\text { II } \\
\text { II }\end{array}$ & $\begin{array}{l}2333.39 \\
2335.42 \\
2335.47 \\
2337.95 \\
2340.39\end{array}$ & $\begin{array}{l}3 ? \\
2 \\
2 ?\end{array}$ & $\begin{array}{c}2 \\
15 h \\
40\end{array}$ & $\begin{aligned} \text { II } \\
\text { I } \\
\text { II } \\
\text { II } \\
\text { I }\end{aligned}$ & $\begin{array}{l}2500.57 \\
2500.89 \\
2501.17 \\
2501.99 \\
2505.46\end{array}$ & $\begin{array}{r}10 \\
5\end{array}$ & $\begin{array}{r}2 \\
1 \\
2 \\
30 \\
10\end{array}$ & \\
\hline $\begin{array}{l}2181.51 \\
2181.85 \\
2182.55 \\
2183.29 \\
2183.61\end{array}$ & & $\begin{array}{l}2 \\
2 \\
3 h \\
7 \\
3 h\end{array}$ & $\begin{array}{l}\text { II } \\
\text { II } \\
\text { II } \\
\text { II } \\
\text { II }\end{array}$ & $\begin{array}{l}2340.46 \\
2340.75 \\
2344.65 \\
2349.39 \\
2350.03\end{array}$ & $\begin{array}{l}5 \\
1\end{array}$ & $\begin{array}{c}8 h \\
3 h \\
20 \\
8 \\
1\end{array}$ & $\begin{array}{l}\text { II } \\
\text { II } \\
\text { II } \\
\text { II } \\
\text { II }\end{array}$ & $\begin{array}{l}2508.04 \\
2509.85 \\
2510.27 \\
2510.50 \\
2512.04\end{array}$ & $\begin{array}{r}1 \\
20\end{array}$ & $\begin{array}{r}2 \\
2 \\
1 \\
5 \\
100\end{array}$ & \\
\hline $\begin{array}{l}2184.78 \\
2185.69 \\
2188.25 \\
2189.42 \\
2193.08\end{array}$ & 60 & $\begin{array}{c}2 \mathrm{Lu} ? \\
100 \\
5 \\
8 \\
2\end{array}$ & $\begin{array}{l}\text { II } \\
\text { II } \\
\text { II } \\
\text { II } \\
\text { II }\end{array}$ & \begin{tabular}{l|}
2361.09 \\
2362.87 \\
2363.48 \\
2365.45 \\
2366.78
\end{tabular} & $\begin{array}{c}10 \\
3\end{array}$ & $\begin{array}{c}6 \\
20 \\
4 h \\
15 \\
12\end{array}$ & $\begin{array}{l}\text { II } \\
\text { II } \\
\text { II } \\
\text { II } \\
\text { II }\end{array}$ & $\begin{array}{l}2512.58 \\
2515.60 \\
2516.36 \\
2516.83 \\
2517.99\end{array}$ & $\begin{array}{l}4 \\
3\end{array}$ & $\begin{array}{c}10 h \\
5 \\
10 \\
30 \\
7\end{array}$ & \\
\hline $\begin{array}{l}2193.40 \\
2195.16 \\
2198.14 \\
2201.19 \\
2203.07\end{array}$ & $-1-1,-1$ & $\begin{array}{c}4 d \\
2 \\
20 h \\
3 ? \\
2 ?\end{array}$ & $\begin{array}{l}\text { II } \\
\text { II } \\
\text { II } \\
\text { II } \\
\text { II }\end{array}$ & $\begin{array}{l}2367.58 \\
2369.44 \\
2369.87 \\
2370.10 \\
2373.06\end{array}$ & 1 & $\begin{array}{l}10 h \\
10 \\
1 ? \\
3 h \\
5\end{array}$ & $\begin{array}{l}\text { II } \\
\text { II } \\
\text { II } \\
\text { II } \\
\text { II }\end{array}$ & $\begin{array}{l}2520.32 \\
2521.02 \\
2522.41 \\
2524.98 \\
2526.28\end{array}$ & $\begin{array}{c}1 \\
8 \\
\end{array}$ & $\begin{array}{r}3 \\
6 \\
15 \\
4 \\
5 h\end{array}$ & \\
\hline
\end{tabular}


TABLE 1.-Arc and spark spectra of ytterbium $(\mathrm{Z}=70)$-Continued

\begin{tabular}{|c|c|c|c|c|c|c|c|c|c|c|c|}
\hline \multirow{2}{*}{$\lambda_{\text {aiz }} \mathbf{A}$} & \multicolumn{2}{|c|}{$\begin{array}{l}\text { Intensity and } \\
\text { character }\end{array}$} & \multirow{2}{*}{$\begin{array}{l}\text { Spec- } \\
\text { trum }\end{array}$} & \multirow{2}{*}{$\lambda$ air $A$} & \multicolumn{2}{|c|}{$\begin{array}{l}\text { Intensity and } \\
\text { character }\end{array}$} & \multirow{2}{*}{$\begin{array}{l}\text { Spec- } \\
\text { trum }\end{array}$} & \multirow{2}{*}{$\lambda$ air $A$} & \multicolumn{2}{|c|}{$\begin{array}{l}\text { Intensity and } \\
\text { character }\end{array}$} & \multirow{2}{*}{$\begin{array}{l}\text { Spec- } \\
\text { trum }\end{array}$} \\
\hline & Arc & Spark & & & Arc & Spark & & & Arc & Spark & \\
\hline $\begin{array}{l}2527.85 \\
2528.32 \\
2529.54 \\
2532.58 \\
2536.01\end{array}$ & \begin{tabular}{l}
2 \\
\hdashline 1
\end{tabular} & $\begin{array}{c}10 \\
7 \\
4 h \\
3 h \\
10\end{array}$ & $\begin{array}{l}\text { II } \\
\text { II } \\
\text { II } \\
\text { II } \\
\text { II }\end{array}$ & $\begin{array}{l}2619.90 \\
2621.13 \\
2621.66 \\
2623.20 \\
2627.04\end{array}$ & $\begin{array}{l}2 \\
2 e \\
1 \\
1\end{array}$ & $\begin{array}{r}5 \\
100 \\
4 \\
5 \\
20\end{array}$ & $\begin{array}{r}\text { II } \\
\text { III } \\
\text { II } \\
\text { II } \\
\text { II }\end{array}$ & $\begin{array}{l}2717.66 \\
2718.35 \\
2719.00 \\
2722.19 \\
2729.06\end{array}$ & $\begin{array}{c}-7 \\
7\end{array}$ & $\begin{array}{c}3 \\
40 \\
2 ? \\
6 \\
1\end{array}$ & $\begin{array}{l}\text { II } \\
\text { II } \\
\text { II } \\
\text { II } \\
\text { II }\end{array}$ \\
\hline $\begin{array}{l}2537.64 \\
2538.18 \\
2538.68 \\
2539.63 \\
2540.61\end{array}$ & $\begin{array}{r}8 \\
2 \\
30 \\
-\end{array}$ & $\begin{array}{r}25 \\
6 \\
40 \\
2 \\
2\end{array}$ & $\begin{array}{l}\text { II } \\
\text { II } \\
\text { II } \\
\text { II } \\
\text { II }\end{array}$ & $\begin{array}{l}2628.04 \\
2628.78 \\
2631.70 \\
2632.66 \\
2634.32\end{array}$ & $\begin{array}{l}1 \\
1 \\
5\end{array}$ & $\begin{array}{r}4 \\
2 \\
4 \\
4 \\
20\end{array}$ & $\begin{array}{l}\text { II } \\
\text { II } \\
\text { II } \\
\text { II } \\
\text { II }\end{array}$ & $\begin{array}{l}2729.49 \\
2731.53 \\
2732.74 \\
2734.08 \\
2736.56\end{array}$ & $\begin{array}{l}8 \\
1 \\
\end{array}$ & $\begin{array}{r}2 \\
1 \\
40 \\
6 \\
3\end{array}$ & $\begin{array}{l}\text { II } \\
\text { II } \\
\text { II } \\
\text { II } \\
\text { II }\end{array}$ \\
\hline $\begin{array}{l}2541.1 \\
2542.82 \\
2545.85 \\
2547.30 \\
2547.47\end{array}$ & 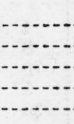 & $\begin{array}{l}3 H \\
8 H \\
2 \\
1 \\
4\end{array}$ & $\begin{array}{l}\text { II } \\
\text { II } \\
\text { II } \\
\text { II } \\
\text { II }\end{array}$ & $\begin{array}{l}2635.39 \\
2636.49 \\
2638.09 \\
2639.44 \\
2640.52\end{array}$ & $\begin{array}{l}1 e \\
5\end{array}$ & $\begin{array}{l}5 h \\
5 h \\
60 \\
15 \\
15 h\end{array}$ & $\begin{array}{r}\text { II } \\
\text { II } \\
\text { III } \\
\text { II } \\
\text { II }\end{array}$ & $\begin{array}{l}2737.61 \\
2738.81 \\
2740.38 \\
2740.78 \\
2741.15\end{array}$ & \begin{tabular}{|c|}
-1 \\
-1
\end{tabular} & $\begin{array}{l}3 \\
2 \\
1 \\
1 \\
1\end{array}$ & $\begin{array}{l}\text { II } \\
\text { II } \\
\text { II }\end{array}$ \\
\hline $\begin{array}{l}2548.06 \\
2548.73 \\
2550.04 \\
2550.42 \\
2550.79\end{array}$ & 2 & $\begin{array}{l}1 h \\
8 \\
4 \\
3 \\
4 h\end{array}$ & $\begin{array}{l}\text { II } \\
\text { II } \\
\text { II } \\
\text { II } \\
\text { II }\end{array}$ & $\begin{array}{l}2641.90 \\
2642.55 \\
2643.65 \\
2644.31 \\
2646.45\end{array}$ & $\begin{array}{l}4 \\
5 e \\
5 \\
2\end{array}$ & $\begin{array}{r}10 \\
150 \\
5 \\
40 \\
10\end{array}$ & $\begin{array}{r}\text { II } \\
\text { III } \\
\text { II } \\
\text { II } \\
\text { II }\end{array}$ & $\begin{array}{l}2741.72 \\
2742.64 \\
2745.11 \\
2745.70 \\
2747.59\end{array}$ & \begin{tabular}{c}
2 \\
\hdashline-1 \\
2
\end{tabular} & $\begin{array}{r}20 \\
1 \\
2 \\
4 \\
20\end{array}$ & $\begin{array}{l}\text { II } \\
\text { II } \\
\text { II } \\
\text { II } \\
\text { II }\end{array}$ \\
\hline $\begin{array}{l}2552.13 \\
2552.69 \\
2555.31 \\
2556.26 \\
2557.24\end{array}$ & $\begin{array}{c}10 \\
8 \\
2\end{array}$ & $\begin{array}{l}40 \\
30 \\
50 h \\
3 \\
8\end{array}$ & $\begin{array}{l}\text { II } \\
\text { II } \\
\text { II } \\
\text { II } \\
\text { II }\end{array}$ & $\begin{array}{l}2647.25 \\
2647.46 \\
2648.80 \\
2649.78 \\
2650.73\end{array}$ & $\begin{array}{l}2 \\
2 \\
4 \\
2\end{array}$ & $\begin{array}{r}4 \\
7 \\
8 \\
10 \\
4\end{array}$ & $\begin{array}{l}\text { II } \\
\text { II } \\
\text { II } \\
\text { II } \\
\text { II }\end{array}$ & $\begin{array}{l}2748.03 \\
2748.65 \\
2749.64 \\
2749.96 \\
2750.47\end{array}$ & $\begin{array}{c}10 \\
50\end{array}$ & $\begin{array}{r}3 \\
40 \\
2 \\
10 \\
200\end{array}$ & $\begin{array}{l}\text { II } \\
\text { II } \\
\text { II } \\
\text { II } \\
\text { II }\end{array}$ \\
\hline $\begin{array}{l}2557.70 \\
2559.03 \\
2559.94 \\
2560.57 \\
2561.64\end{array}$ & & $\begin{array}{l}4 \\
1 \\
2 \\
5 \\
1\end{array}$ & $\begin{array}{l}\text { II } \\
\text { II } \\
\text { II } \\
\text { II } \\
\text { II }\end{array}$ & $\begin{array}{l}2651.72 \\
2652.23 \\
2653.74 \\
2656.11 \\
2659.27\end{array}$ & $\begin{array}{c}2 e \\
2 e \\
50 \\
2 \\
2\end{array}$ & $\begin{array}{r}60 \\
60 \\
200 \\
7 \\
5\end{array}$ & $\begin{array}{c}\text { III } \\
\text { III } \\
\text { II } \\
\text { II } \\
\text { II }\end{array}$ & $\begin{array}{l}2751.44 \\
2753.67 \\
2754.93 \\
2756.02 \\
2756.79\end{array}$ & $\begin{array}{c}3 \\
\\
\\
\end{array}$ & $\begin{array}{c}20 \\
3 h \\
4 \\
4 \\
3 ?\end{array}$ & $\begin{array}{l}\text { II } \\
\text { II } \\
\text { II } \\
\text { II } \\
\text { II }\end{array}$ \\
\hline $\begin{array}{l}2565.56 \\
2566.80 \\
2567.63 \\
2568.17 \\
2571.34\end{array}$ & $10 e$ & $\begin{array}{c}15 \\
3 \\
300 \\
2 h \\
20\end{array}$ & $\begin{array}{r}\text { II } \\
\text { II } \\
\text { III } \\
\text { II } \\
\text { II }\end{array}$ & $\begin{array}{l}2660.01 \\
2661.86 \\
2665.02 \\
2666.11 \\
2666.98\end{array}$ & $\begin{array}{c}10 \\
5 e \\
5 e\end{array}$ & $\begin{array}{r}6 \\
1 \\
60 \\
150 \\
150\end{array}$ & $\begin{array}{r}\text { II } \\
\text { II } \\
\text { II } \\
\text { III } \\
\text { III }\end{array}$ & $\begin{array}{l}2758.99 \\
2759.54 \\
2760.78 \\
2761.38 \\
2764.42\end{array}$ & $\begin{array}{c}1 \\
-3 \\
3 \\
1\end{array}$ & $\begin{array}{r}10 \\
4 \\
25 \\
25 \\
10\end{array}$ & $\begin{array}{l}\text { II } \\
\text { II } \\
\text { II } \\
\text { II } \\
\text { II }\end{array}$ \\
\hline $\begin{array}{l}2572.11 \\
2573.13 \\
2574.78 \\
2577.68 \\
2579.58\end{array}$ & $\begin{array}{l}3 \\
1 \\
2 \\
5 e\end{array}$ & $\begin{array}{r}3 \\
10 \\
3 \\
30 \\
200\end{array}$ & $\begin{array}{l}\text { II } \\
\text { II } \\
\text { II } \\
\text { II } \\
\text { III }\end{array}$ & $\begin{array}{l}2668.75 \\
2671.98 \\
2672.65 \\
2673.37 \\
2674.87\end{array}$ & $\begin{array}{r}2 \\
10 \\
20 \\
\end{array}$ & $\begin{array}{r}20 \\
2 \\
80 \\
2 \\
4\end{array}$ & $\begin{array}{l}\text { II } \\
\text { I } \\
\text { II } \\
\text { II } \\
\text { II }\end{array}$ & $\begin{array}{l}2765.04 \\
2765.54 \\
2768.27 \\
2771.34 \\
2774.32\end{array}$ & 3 & $\begin{array}{r}1 \\
7 \\
2 \\
30 \\
4\end{array}$ & $\begin{array}{l}\text { II } \\
\text { II } \\
\text { II } \\
\text { II } \\
\text { II }\end{array}$ \\
\hline $\begin{array}{l}2583.72 \\
2586.28 \\
2588.65 \\
2591.02 \\
2592.71\end{array}$ & 1 & $\begin{array}{l}2 \\
4 \\
8 h \\
5 \\
3\end{array}$ & $\begin{array}{l}\text { II } \\
\text { II } \\
\text { II } \\
\text { II } \\
\text { II }\end{array}$ & $\begin{array}{l}2676.13 \\
2677.37 \\
2678.60 \\
2680.40 \\
2683.42\end{array}$ & & $\begin{array}{c}2 \\
40 \\
1 ? \\
3 \\
10\end{array}$ & $\begin{array}{l}\text { II } \\
\text { II } \\
\text { II } \\
\text { II } \\
\text { II }\end{array}$ & $\begin{array}{l}2775.45 \\
2776.28 \\
2780.03 \\
2782.21 \\
2782.57\end{array}$ & \begin{tabular}{c}
-10 \\
\hdashline \\
\hdashline
\end{tabular} & $\begin{array}{r}2 \\
50 \\
3 \\
1 \\
1\end{array}$ & $\begin{array}{l}\text { II } \\
\text { II } \\
\text { II } \\
\text { II } \\
\text { II }\end{array}$ \\
\hline $\begin{array}{l}2594.18 \\
2594.48 \\
2596.15 \\
2596.29 \\
2596.73\end{array}$ & $\begin{array}{l}1 \\
2 \\
2 \\
2 \\
2 \\
2\end{array}$ & $\begin{array}{l}5 \\
5 \\
6 \\
7 \\
3\end{array}$ & $\begin{array}{l}\text { II } \\
\text { II } \\
\text { II } \\
\text { II } \\
\text { II }\end{array}$ & $\begin{array}{l}2684.75 \\
2685.99 \\
2687.95 \\
2690.99 \\
2691.99\end{array}$ & $\begin{array}{r}3 \\
\\
\\
\\
\end{array}$ & $\begin{array}{r}25 \\
3 \\
5 \\
40 \\
6\end{array}$ & $\begin{array}{l}\text { II } \\
\text { II } \\
\text { II } \\
\text { II } \\
\text { II }\end{array}$ & $\begin{array}{l}2784.66 \\
2785.80 \\
2787.00 \\
2787.98 \\
2788.31\end{array}$ & $\begin{array}{r}3 \\
\\
\\
\\
\end{array}$ & $\begin{array}{r}30 \\
2 \\
1 \\
4 \\
10\end{array}$ & $\begin{array}{l}\text { II } \\
\text { II } \\
\text { II } \\
\text { II } \\
\text { II }\end{array}$ \\
\hline $\begin{array}{l}2597.26 \\
2599.16 \\
2600.19 \\
2600.84 \\
2602.41\end{array}$ & $\begin{array}{l}1 \\
2 \\
1 \\
1\end{array}$ & $\begin{array}{r}20 \\
50 \\
3 \\
3 \\
1\end{array}$ & $\begin{array}{l}\text { II } \\
\text { II } \\
\text { II } \\
\text { II } \\
\text { II }\end{array}$ & $\begin{array}{l}2692.40 \\
2692.69 \\
2694.61 \\
2695.43 \\
2696.63\end{array}$ &.- & $\begin{array}{l}2 \\
2 \\
1 ? \\
9 \\
6\end{array}$ & $\begin{array}{l}\text { II } \\
\text { II } \\
\text { II } \\
\text { II } \\
\text { II }\end{array}$ & $\begin{array}{l}2789.44 \\
2791.58 \\
2793.28 \\
2794.44 \\
2794.78\end{array}$ & $\begin{array}{r}-1 \\
1 \\
1 \\
-\end{array}$ & $\begin{array}{r}3 \\
1 \\
10 \\
7 \\
4\end{array}$ & $\begin{array}{l}\text { II } \\
\text { II } \\
\text { II } \\
\text { II } \\
\text { II }\end{array}$ \\
\hline $\begin{array}{l}2604.04 \\
2607.86 \\
2608.45 \\
2609.12 \\
2610.86\end{array}$ & $\begin{array}{c}4 \\
3 \\
-\end{array}$ & $\begin{array}{r}10 \\
7 \\
2 \\
4 \\
20\end{array}$ & $\begin{array}{l}\text { II } \\
\text { II } \\
\text { II } \\
\text { II } \\
\text { II }\end{array}$ & $\begin{array}{l}2700.80 \\
2704.52 \\
2708.08 \\
2708.84 \\
2709.72\end{array}$ & 1 & $\begin{array}{r}10 \\
3 \\
1 \\
5 \\
2\end{array}$ & $\begin{array}{l}\text { II } \\
\text { II } \\
\text { II } \\
\text { II } \\
\text { II }\end{array}$ & $\begin{array}{l}2795.10 \\
2795.62 \\
2797.79 \\
2798.21 \\
2799.39\end{array}$ & {$\left[\begin{array}{c}- \\
\hdashline \\
\hdashline\end{array}\right.$} & $\begin{array}{r}4 \\
10 \\
8 \\
10 \\
3\end{array}$ & $\begin{array}{l}\text { II } \\
\text { I } \\
\text { II } \\
\text { II } \\
\text { II }\end{array}$ \\
\hline $\begin{array}{l}2612.03 \\
2612.60 \\
2615.25 \\
2617.00 \\
2619.06\end{array}$ & $\begin{array}{l}1 \\
1 \\
5 \\
6 \\
2\end{array}$ & $\begin{array}{r}4 \\
4 \\
20 \\
25 \\
6\end{array}$ & $\begin{array}{l}\text { II } \\
\text { II } \\
\text { II } \\
\text { II } \\
\text { II }\end{array}$ & $\begin{array}{l}2710.56 \\
2712.66 \\
2714.42 \\
2715.94 \\
2717.19\end{array}$ & $\begin{array}{r}3 \\
3 \\
\\
\end{array}$ & $\begin{array}{c}15 d \\
7 \\
5 \\
2 \\
2 h\end{array}$ & $\begin{array}{l}\text { II } \\
\text { II } \\
\text { II } \\
\text { II } \\
\text { II }\end{array}$ & $\begin{array}{l}2800.04 \\
2803.44 \\
2804.27 \\
2806.05 \\
2807.22\end{array}$ & a & $\begin{array}{c}15 \\
80 \\
4 \\
1 \\
3 d\end{array}$ & $\left\{\begin{array}{r}\text { II } \\
\text { II } \\
\text { IIII } \\
\text { II } \\
\text { I? } \\
\text { II }\end{array}\right.$ \\
\hline
\end{tabular}


TABLE 1.-Arc and spark spectra of ytterbium $(\mathrm{Z}=70)$-Continued

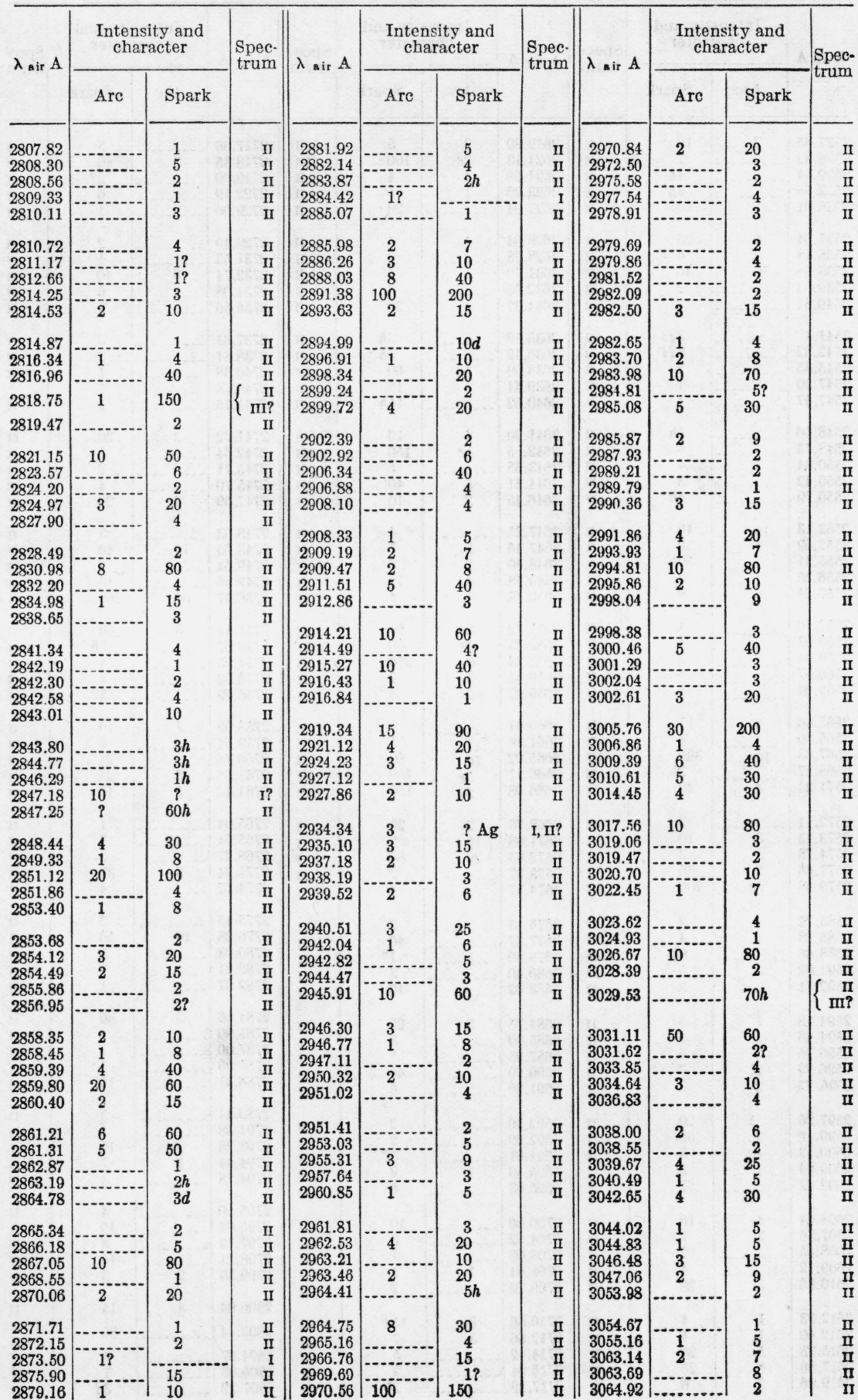


TABLE 1.-Arc and spark spectra of ytterbium $(\mathrm{Z}=70)$-Continued

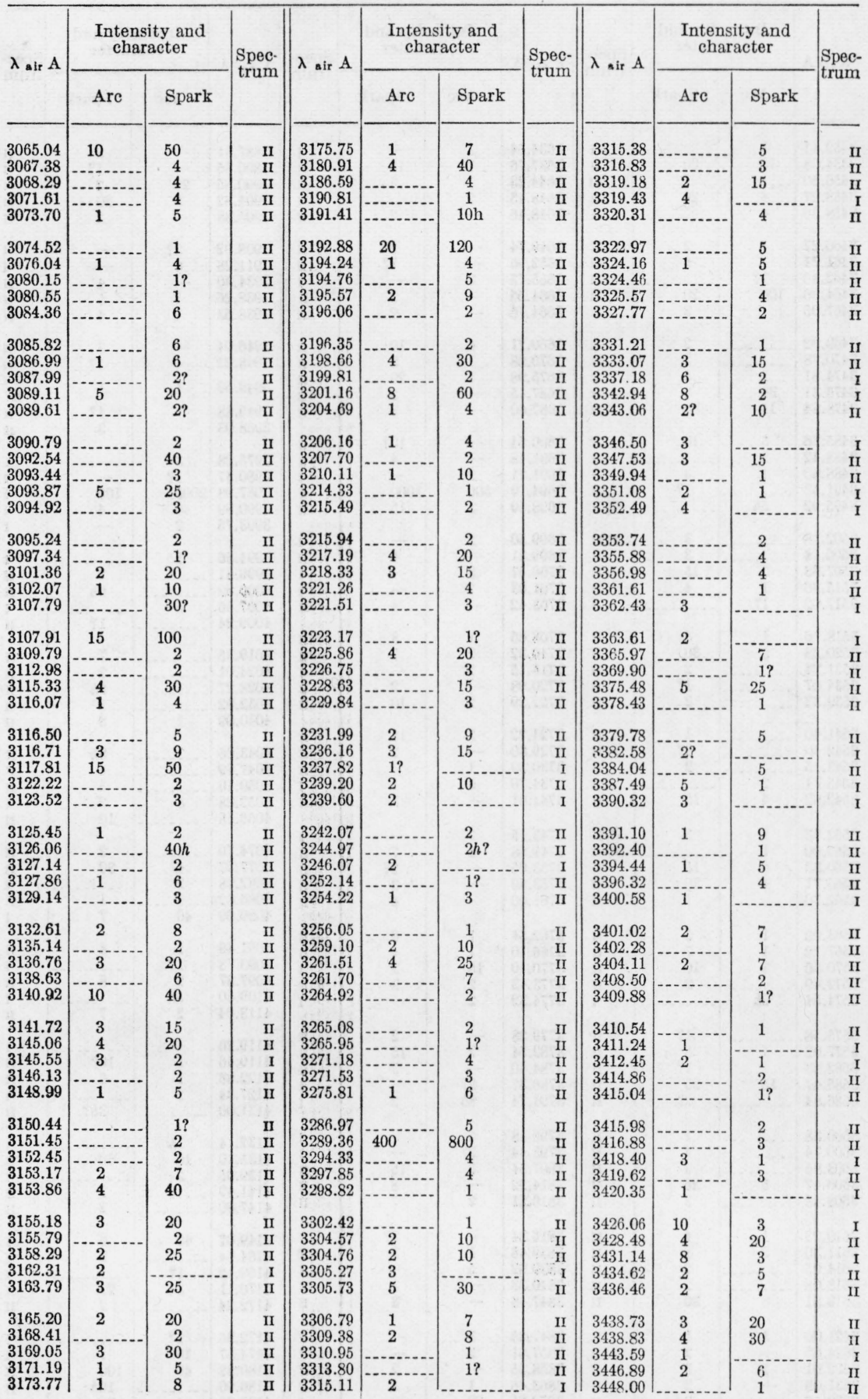


TABLE 1.-Arc and spark spectra of ytterbium $(\mathrm{Z}=70)$-Continued

\begin{tabular}{|c|c|c|c|c|c|c|c|c|c|c|c|}
\hline \multirow{2}{*}{$\lambda=$ ir $\mathbf{A}$} & \multicolumn{2}{|c|}{$\begin{array}{l}\text { Intensity and } \\
\text { character }\end{array}$} & \multirow{2}{*}{$\begin{array}{l}\text { Spec- } \\
\text { trum }\end{array}$} & \multirow{2}{*}{$\lambda$. ir $A$} & \multicolumn{2}{|c|}{$\begin{array}{l}\text { Intensity and } \\
\text { character }\end{array}$} & \multirow{2}{*}{$\begin{array}{l}\text { Spec- } \\
\text { trum }\end{array}$} & \multirow{2}{*}{$\lambda_{\text {nir }} \mathrm{A}$} & \multicolumn{2}{|c|}{$\begin{array}{l}\text { Intensity and } \\
\text { character }\end{array}$} & \multirow{2}{*}{$\begin{array}{l}\text { Spec- } \\
\text { trumm }\end{array}$} \\
\hline & Arc & Spark & & & Arc & Spark & & & Arc & Spark & \\
\hline $\begin{array}{l}3452.41 \\
3454.08 \\
3456.30 \\
3458.27 \\
3458.36\end{array}$ & \begin{tabular}{c}
3 \\
10 \\
\hdashline 4 \\
4
\end{tabular} & $\begin{array}{l}60 \\
1 ? \\
20\end{array}$ & $\begin{array}{l}\text { I } \\
\text { II } \\
\text { II } \\
\text { II } \\
\text { I }\end{array}$ & $\begin{array}{l}3634.54 \\
3637.76 \\
3644.23 \\
3648.13 \\
3648.46\end{array}$ & $\begin{array}{l}3 \\
7 \\
1 \\
2 \\
-\end{array}$ & $\begin{array}{r}\overline{15} \\
3 \\
2\end{array}$ & $\begin{array}{l}\text { I } \\
\text { II } \\
\text { II } \\
\text { I } \\
\text { II }\end{array}$ & $\begin{array}{l}3887.31 \\
3890.86 \\
3900.86 \\
3904.82 \\
3905.88\end{array}$ & $\begin{array}{l}\overline{-} \\
20 \\
3 \\
-\end{array}$ & $\begin{array}{l}8 \\
1 ? \\
2 \\
20 \\
2\end{array}$ & $\begin{array}{l}\text { II } \\
\text { II } \\
\text { I } \\
\text { II } \\
\text { II }\end{array}$ \\
\hline $\begin{array}{l}3460.27 \\
3461.71 \\
3462.36 \\
3464.36 \\
3467.05\end{array}$ & $\begin{array}{c}7 \\
1 \\
1 ? \\
100 \\
\end{array}$ & $\begin{array}{r}20 \\
3\end{array}$ & $\begin{array}{r}\text { I } \\
\text { II } \\
\text { I } \\
\text { I } \\
\text { II }\end{array}$ & $\begin{array}{l}3649.74 \\
3652.96 \\
3655.73 \\
3664.56 \\
3664.76\end{array}$ & $\begin{array}{l}\overline{-} \\
\frac{3}{-}\end{array}$ & $\begin{array}{l}2 \\
1 ? \\
-2 \\
2\end{array}$ & $\begin{array}{l}\text { II } \\
\text { II } \\
\text { I } \\
\text { II } \\
\text { II }\end{array}$ & $\begin{array}{l}3908.02 \\
3911.28 \\
3934.30 \\
3938.26 \\
3938.53\end{array}$ & $\begin{array}{l}2 ? \\
\frac{6}{1} \\
1 \\
1\end{array}$ & $\begin{array}{l}- \\
4 \\
5 \\
4\end{array}$ & $\begin{array}{l}\text { I } \\
\text { I } \\
\text { II } \\
\text { II } \\
\text { II }\end{array}$ \\
\hline $\begin{array}{l}3469.92 \\
3470.78 \\
3474.81 \\
3476.31 \\
3478.84\end{array}$ & $\begin{array}{r}2 \\
30 \\
15\end{array}$ & $\begin{array}{r}2 \\
2 \\
5 \\
8 \\
80\end{array}$ & $\begin{array}{l}\text { II } \\
\text { II } \\
\text { II } \\
\text { I } \\
\text { II }\end{array}$ & $\begin{array}{l}3669.71 \\
3670.68 \\
3675.08 \\
3687.15 \\
3687.60\end{array}$ & $\begin{array}{r}6 \\
1 \\
5 \\
- \\
-\end{array}$ & $\begin{array}{r}10 \\
8 \\
20 \\
2 \\
3\end{array}$ & $\begin{array}{l}\text { II } \\
\text { II } \\
\text { II } \\
\text { II } \\
\text { II }\end{array}$ & $\begin{array}{l}3946.94 \\
3948.23 \\
3949.59 \\
3949.88 \\
3968.03\end{array}$ & $\begin{array}{l}- \\
1 \\
=\end{array}$ & $\begin{array}{l}3 \\
1 ? \\
1 \\
1 ? \\
3\end{array}$ & $\left\{\begin{array}{r}\text { II } \\
\text { II } \\
\text { I? } \\
\text { II } \\
\text { II }\end{array}\right.$ \\
\hline $\begin{array}{l}3485.76 \\
3488.42 \\
3488.80 \\
3491.63 \\
3495.92\end{array}$ & $\begin{array}{l}3 \\
3 \\
1 \\
5 \bar{h}\end{array}$ & $\begin{array}{l}10 \\
3 \\
1 ? \\
0\end{array}$ & $\begin{array}{r}\text { II } \\
\text { I } \\
\text { II } \\
\text { II } \\
\text { I }\end{array}$ & $\begin{array}{l}3690.54 \\
3691.48 \\
3691.71 \\
3694.19 \\
3698.59\end{array}$ & $\begin{array}{c}\bar{z} \\
500 \\
3\end{array}$ & $\begin{array}{c}10 ? \\
\frac{4}{1000} \\
15\end{array}$ & $\begin{array}{l}\text { II } \\
\text { II } \\
\text { I } \\
\text { II } \\
\text { II }\end{array}$ & $\begin{array}{l}3975.28 \\
3980.37 \\
3987.98 \\
3990.89 \\
3903.75\end{array}$ & $\begin{array}{c}3 \\
1 ? \\
2000 \\
40\end{array}$ & $\begin{array}{r}- \\
100 \\
4\end{array}$ & $\begin{array}{l}\text { I } \\
\text { I } \\
\text { I } \\
\text { I }\end{array}$ \\
\hline $\begin{array}{l}3502.18 \\
3506.14 \\
3507.83 \\
3515.86 \\
3517.02\end{array}$ & $\begin{array}{l}3 \\
1 \\
1 ?\end{array}$ & $\begin{array}{r}3 \\
3 \\
15 \\
4 \\
\end{array}$ & $\begin{array}{l}\text { II } \\
\text { II } \\
\text { II } \\
\text { II } \\
\text { I }\end{array}$ & $\begin{array}{l}3699.50 \\
3699.81 \\
\mathbf{3 7 0 0 . 5 7} \\
\mathbf{3 7 0 1 . 0 3} \\
\mathbf{3 7 0 3 . 4 2}\end{array}$ & $\begin{array}{l}\frac{2}{3} \\
2 ? \\
-\end{array}$ & $\frac{\overline{4}}{\overline{4}}$ & $\begin{array}{r}\text { I } \\
\text { II } \\
\text { I } \\
\text { I } \\
\text { II }\end{array}$ & $\begin{array}{l}3994.86 \\
3999.81 \\
4000.89 \\
4007.36 \\
000.94\end{array}$ & 5 & $\begin{array}{c}2 \\
6 h \\
\end{array}$ & II \\
\hline $\begin{array}{l}3518.16 \\
3520.28 \\
3531.24 \\
3534.07 \\
3539.33\end{array}$ & \begin{tabular}{c}
1 \\
5 \\
\\
\hdashline \\
\end{tabular} & $\begin{array}{c}5 \\
30 \\
2 \\
1 ? \\
2\end{array}$ & $\begin{array}{l}\text { II } \\
\text { II } \\
\text { II } \\
\text { II } \\
\text { II }\end{array}$ & $\begin{array}{l}3708.66 \\
3710.32 \\
3716.15 \\
3720.98 \\
3722.29\end{array}$ & $\begin{array}{l}1 \\
2 \\
2 ? \\
\frac{2}{2}\end{array}$ & $\begin{array}{r}3 \\
7 \\
2 \\
10\end{array}$ & $\begin{array}{l}\text { II } \\
\text { II } \\
\text { I } \\
\text { II } \\
\text { II }\end{array}$ & $\begin{array}{l}4019.35 \\
4024.01 \\
4028.27 \\
4032.92 \\
4040.09\end{array}$ & - & $\begin{array}{l}7 \\
2 \\
8 h \\
1 \\
8\end{array}$ & $\begin{array}{l}\text { II } \\
\text { II } \\
\text { II } \\
\text { II }\end{array}$ \\
\hline $\begin{array}{l}3541.40 \\
3542.40 \\
3543.15 \\
3545.71 \\
3549.82\end{array}$ & \begin{tabular}{c}
-1 \\
2 \\
\hdashline
\end{tabular} & $\begin{array}{l}1 \\
2 ? \\
2 \\
15\end{array}$ & $\begin{array}{l}\text { II } \\
\text { II } \\
\text { II } \\
\text { I } \\
\text { II }\end{array}$ & $\begin{array}{l}3724.22 \\
3729.80 \\
3730.39 \\
3734.70 \\
3741.61\end{array}$ & $\frac{3}{1}$ & $\begin{array}{r}15 \\
2 \\
3 \\
1 \\
3\end{array}$ & $\begin{array}{l}\text { II } \\
\text { II } \\
\text { II } \\
\text { I } \\
\text { II }\end{array}$ & $\begin{array}{l}4043.06 \\
4047.39 \\
4050.10 \\
4052.28 \\
4056.18\end{array}$ & $\begin{array}{c} \\
8 \\
-\end{array}$ & $\begin{array}{r}0 \\
8 h \\
4 \\
3 \\
2 \\
10\end{array}$ & $\begin{array}{l}\text { II } \\
\text { II } \\
\text { II } \\
\text { I } \\
\text { II }\end{array}$ \\
\hline $\begin{array}{l}3552.33 \\
3559.00\end{array}$ & $\begin{array}{l}1 \\
1\end{array}$ & 3 & $\begin{array}{r}\text { II } \\
\text { I }\end{array}$ & $\begin{array}{l}3743.15 \\
3749.68\end{array}$ & $\begin{array}{l}2 ? \\
2\end{array}$ & $\overline{7}$ & I & 4074.70 & & 2 & II \\
\hline $\begin{array}{l}3560.33 \\
3560.71\end{array}$ & $\begin{array}{l}6 \\
4\end{array}$ & $\begin{array}{l}15 \\
30\end{array}$ & $\begin{array}{l}\text { II } \\
\text { II }\end{array}$ & $\begin{array}{l}3753.05 \\
3753.89\end{array}$ & \pm & $2 ?$ & II & $\begin{array}{l}4077.27 \\
4082.98\end{array}$ & 2 & 30 & II \\
\hline 3562.70 & & 1 & II & 3761.00 & - & 4 & II & $\begin{array}{l}4086.62 \\
4089.69\end{array}$ & & $\frac{1}{7}$ & II \\
\hline $\begin{array}{l}3563.93 \\
3567.12 \\
3570.56\end{array}$ & $\begin{array}{l}1 \\
1 \\
3\end{array}$ & $\begin{array}{r}5 \\
7 \\
10\end{array}$ & $\begin{array}{l}\text { II } \\
\text { II } \\
\text { II }\end{array}$ & $\begin{array}{l}3762.54 \\
3766.10 \\
3770.10\end{array}$ & $\begin{array}{r}1 \\
1 \\
15\end{array}$ & $\begin{array}{l}6 \\
4 \\
3\end{array}$ & II & $\begin{array}{l}4091.49 \\
4093.73\end{array}$ & $\begin{array}{l}1 \\
1 ?\end{array}$ & 4 & $\begin{array}{l}\text { II } \\
\text { I }\end{array}$ \\
\hline & & 2 & II & $\begin{array}{l}3770.10 \\
3773.43\end{array}$ & $\begin{array}{r}10 \\
2\end{array}$ & $\begin{array}{l}0 \\
3\end{array}$ & II & 4097.87 & 2 & 8 & II \\
\hline 3574.54 & $2 h$ & 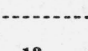 & I & 3774.32 & 2 & - & I & $\begin{array}{l}4109.60 \\
4113.04\end{array}$ & $\begin{array}{l}5 h \\
2\end{array}$ & 7 & I \\
\hline $\begin{array}{l}3575.58 \\
3577.02 \\
3582.83 \\
3585.47 \\
3586.84\end{array}$ & $\begin{array}{c}1 \\
15 \\
15\end{array}$ & $\begin{array}{c}1 ? \\
5 \\
1 ? \\
25 \\
2 ?\end{array}$ & $\begin{array}{l}\text { II } \\
\text { II } \\
\text { II } \\
\text { II } \\
\text { II }\end{array}$ & $\begin{array}{l}3779.28 \\
3782.54 \\
3784.40 \\
3786.37 \\
3791.74\end{array}$ & $\begin{array}{c}\overline{2} \\
\overline{2} ? \\
8 h\end{array}$ & $\begin{array}{r}2 \\
15 \\
3 \\
2\end{array}$ & \begin{tabular}{r|}
$\mathbf{I}$ \\
$\mathbf{I}$ \\
$\mathbf{I}$ \\
$\mathbf{I}$ \\
$\mathbf{I}$
\end{tabular} & $\begin{array}{l}4119.26 \\
4119.46 \\
4122.86 \\
4127.34 \\
4131.00\end{array}$ & $\begin{array}{l}4 \\
5 \\
1 \\
2\end{array}$ & $\begin{array}{c}10 ? \\
6 \\
3 h ?\end{array}$ & $\begin{array}{l}\text { I } \\
\text { II } \\
\text { II } \\
\text { I } \\
\text { II }\end{array}$ \\
\hline $\begin{array}{l}3600.38 \\
3600.74 \\
3603.85 \\
3606.47 \\
3608.48\end{array}$ & 5 & $\begin{array}{r}1 \\
4 \\
3 \\
25 \\
3\end{array}$ & $\begin{array}{l}\text { II } \\
\text { II } \\
\text { II } \\
\text { II } \\
\text { II }\end{array}$ & $\begin{array}{l}3798.18 \\
3798.44 \\
3807.54 \\
3814.22 \\
3816.21\end{array}$ & $\begin{array}{l}2 \\
2 ? \\
3 \\
2\end{array}$ & $\begin{array}{r}- \\
15 \\
8 \\
7\end{array}$ & $\begin{array}{l}\text { I } \\
\text { I } \\
\text { II } \\
\text { II } \\
\text { II }\end{array}$ & $\begin{array}{l}4132.14 \\
4135.10 \\
4139.05 \\
414.49 \\
4147.80\end{array}$ & \begin{tabular}{c}
$3 h$ \\
10 \\
2 \\
\hdashline \\
\hdashline..- \\
\end{tabular} & $\begin{array}{r}60 \\
2 \\
2\end{array}$ & $\begin{array}{l}\text { I } \\
\text { II } \\
\text { I } \\
\text { II } \\
\text { II }\end{array}$ \\
\hline $\begin{array}{l}3610.23 \\
3611.30 \\
3614.05 \\
3618.08 \\
3619.81\end{array}$ & \begin{tabular}{c}
1 \\
2 \\
\hdashline 8
\end{tabular} & $\begin{array}{r}4 \\
8 \\
2 \\
2 \\
30\end{array}$ & $\begin{array}{l}\text { II } \\
\text { II } \\
\text { II } \\
\text { II } \\
\text { II }\end{array}$ & $\begin{array}{l}\mathbf{3 8 1 6 . 3 4} \\
\mathbf{3 8 3 9 . 4 6} \\
\mathbf{3 8 3 9 . 9 2} \\
\mathbf{3 8 4 0 . 3 3} \\
\mathbf{3 8 4 7 . 4 8}\end{array}$ & $\begin{array}{l}-1 \\
5 \\
-\end{array}$ & $\begin{array}{l}2 ? \\
\frac{3}{3} \\
3 \\
3\end{array}$ & \begin{tabular}{l|} 
II \\
II \\
I \\
II \\
II
\end{tabular} & $\begin{array}{l}4149.07 \\
4164.64 \\
4169.12 \\
4170.11 \\
4172.24\end{array}$ & $\begin{array}{c}40 \\
1 ? \\
3\end{array}$ & $\begin{array}{r}6 \\
2 \\
-20 \\
\hdashline 2\end{array}$ & $\begin{array}{l}\text { I } \\
\text { II } \\
\text { I } \\
\text { II } \\
\text { II }\end{array}$ \\
\hline $\begin{array}{l}3621.00 \\
3624.65 \\
3629.91 \\
3631.08 \\
3632.56\end{array}$ & $\frac{1}{1}$ & $\begin{array}{l}5 \\
2 \\
3 \\
2 \\
3 d\end{array}$ & $\begin{array}{l}\text { II } \\
\text { II } \\
\text { II } \\
\text { II } \\
\text { II }\end{array}$ & $\begin{array}{l}3847.86 \\
3857.61 \\
3858.55 \\
3863.46 \\
3872.85\end{array}$ & $\begin{array}{l}3 \\
\frac{3}{1} \\
20\end{array}$ & $\begin{array}{l}\overline{-} \\
\overline{2} \\
5 \\
2\end{array}$ & \begin{tabular}{r|} 
I \\
I \\
II \\
I \\
I
\end{tabular} & $\begin{array}{l}4172.36 \\
4174.57 \\
4180.82 \\
4186.90 \\
4190.30\end{array}$ & \begin{tabular}{c}
$1 ?$ \\
10 \\
40 \\
\hdashline 4
\end{tabular} & $\begin{array}{c}1 \\
100 \\
10 h \\
30\end{array}$ & $\begin{array}{l}\text { I } \\
\text { I } \\
\text { II } \\
\text { II } \\
\text { II }\end{array}$ \\
\hline
\end{tabular}


TABLE 1.-Arc and spark spectra of ytterbium $(\mathrm{Z}=70)$-Continued

\begin{tabular}{|c|c|c|c|c|c|c|c|c|c|c|c|}
\hline \multirow{2}{*}{$\lambda_{\text {sir }} \mathbf{A}$} & \multicolumn{2}{|c|}{$\begin{array}{l}\text { Intensity and } \\
\text { character }\end{array}$} & \multirow{2}{*}{$\begin{array}{l}\text { Spec- } \\
\text { trum }\end{array}$} & \multirow{2}{*}{$\lambda$ =ir $\mathbf{A}$} & \multicolumn{2}{|c|}{$\begin{array}{l}\text { Intensity and } \\
\text { character }\end{array}$} & \multirow{2}{*}{$\begin{array}{l}\text { Spec- } \\
\text { trum }\end{array}$} & \multirow{2}{*}{$\lambda=$ ir $A$} & \multicolumn{2}{|c|}{$\begin{array}{l}\text { Intensity and } \\
\text { character }\end{array}$} & \multirow{2}{*}{$\begin{array}{l}\text { Spec- } \\
\text { trum }\end{array}$} \\
\hline & Arc & Spark & & & Arc & Spark & & & Arc & Spark & \\
\hline 4210.30 & 4 & & I & 4472.43 & $20 h$ & & I & $\mathbf{4 7 4 3 . 3 8}$ & $1 ?$ & & I \\
\hline $\begin{array}{r}4216.72 \\
4218.55\end{array}$ & 4 & $\begin{array}{r}5 \\
50\end{array}$ & II & $\begin{array}{l}4480.27 \\
4482.44\end{array}$ & 10 & $\begin{array}{l}2 \\
2\end{array}$ & II & $\begin{array}{l}\mathbf{4 7 4 5 . 5 2} \\
4746.70\end{array}$ & & $\underset{15 h}{2}$ & $\begin{array}{l}\text { II } \\
\text { II }\end{array}$ \\
\hline 4218.64 & $\begin{array}{r}* \\
15\end{array}$ & $?$ & I & 4484.43 & 10 & $3 h$ & II & $\begin{array}{l}4740.00 \\
4750.01\end{array}$ & & $4 h$ & II \\
\hline 4224.19 & & 1 & II & 4487.27 & 2 & 10 & $\overline{\mathbf{I}}$ & 4751.77 & 4 & & I \\
\hline 4227.94 & & 10 & II & 4488.28 & 4 & & $I$ & $\mathbf{4 7 5 2 . 9 3}$ & 3 & $20 h$ & II \\
\hline 29. & 2 & & I & 4493.96 & 2 & 10 & II & 4758.32 & 2 & 1 & I \\
\hline 4230. & 10 & 4 & II & 4503.51 & & $2 h ?$ & II & $\mathbf{4 7 6 1 . 1 6}$ & & $5 h$ & II \\
\hline $\begin{array}{r}4231.99 \\
4233.45\end{array}$ & $\begin{array}{c}10 \\
2 ?\end{array}$ & 2 & I & $\begin{array}{l}4503.62 \\
\end{array}$ & $\begin{array}{l}5 \\
6\end{array}$ & 1 & 1 & 4765.37 & $3 h$ & 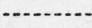 & I \\
\hline & & & & & & & & & & & \\
\hline $\begin{array}{r}4234.55 \\
4244.21\end{array}$ & 2 & 15 & II & 4514.04 & & 2? & II & $\$ 774.61$ & $3 h$ & & I \\
\hline 4247.8 & 2 & $2 h$ & II & & 30 & $\begin{array}{c}100 \\
1 ?\end{array}$ & II & 4774.84 & $3 h$ & $\cdots$ & I \\
\hline & 4 & i & I & $\mathbf{4 5 2 9 . 9 0}$ & 20 & 2 & 1 & 4781.89 & 200 & 5 & I \\
\hline 4252.5 & 5 & 20 & II & $\mathbf{4 5 3 3 . 5 0}$ & 4 & 1 & I & 4784.52 & 3 & & I \\
\hline 4254.78 & 2 & 4 & II & 4536.80 & & $2 ?$ & I & 4785.34 & 2 & & I \\
\hline & 3 & 8 & II & & 17 & 2 & II & & 100 & 500 & II \\
\hline $\begin{array}{r}4256.77 \\
4257.66\end{array}$ & $3^{3 n}$ & 15 & II & $\begin{array}{r}4539.70 \\
4541.35\end{array}$ & $1 ?$ & 6 & II & $\begin{array}{l}4797.85 \\
4808.51\end{array}$ & 1 & $4 h$ & II \\
\hline 4266.99 & & $8 h$ & II & 4543.90 & & 4 & II & $\mathbf{4 8 0 9 . 4 7}$ & & 2 & II \\
\hline 4270.55 & & $3 h$ & II & 4547.01 & & 1 & II & 4812.91 & $3 h$ & & I \\
\hline & 2 & & I & & 2 & 2 & $\mathrm{I}, \mathrm{II} ?$ & 4816.40 & $40 h$ & 1 & I \\
\hline 4273. & & 3 & II & 4553.58 & 10 & 40 & II & & 3 & 30 & II \\
\hline 4277.73 & & 2 & I & 4557.94 & 2 & 6 & II & 4820.25 & 20 & 80 & II \\
\hline 4283.01 & $7 \mathrm{Ca}$ ? & & I & 4564.00 & 50 & 2 & I & 4830.70 & 2 & & I \\
\hline 4300.99 & 6 & 1 & 1 & 4565.99 & & $2 h ?$ & II & 4831.31 & 2 & & I \\
\hline & 2 & & I & 4567.38 & 5 & 1 & 1 & & & $2 h$ & II \\
\hline $\begin{array}{l}4305.9 \\
4306.4\end{array}$ & $20 h$ & 3 & $\mathbf{I}$ & 4568.89 & $2 h$ & 10 & I & 4931.96 & $3 h$ & & 1 \\
\hline $\begin{array}{l}4306.49 \\
4309.82\end{array}$ & 8 & 3 & $\begin{array}{r}\text { II } \\
\text { I }\end{array}$ & $\begin{array}{l}4576.21 \\
4580.75\end{array}$ & $\begin{array}{r}200 \\
2\end{array}$ & 10 & $\begin{array}{l}1 \\
1\end{array}$ & $\begin{array}{l}4834.72 \\
4836.96\end{array}$ & & $\begin{array}{c}10 h \\
120\end{array}$ & $\begin{array}{l}\text { II } \\
\text { II }\end{array}$ \\
\hline 4312.36 & 2 & & I & $\mathbf{4 5 8 2 . 3 6}$ & 50 & 5 & I & 4837.47 & 20 & 1 & I \\
\hline 4316. & 10 & 40 & II & & & $2 h$ & II & 4841.18 & 2 & 2 & $\left\{\begin{array}{l}\mathrm{I} \\
\mathrm{r}\end{array}\right.$ \\
\hline 4322.2 & 3 & $\begin{array}{r}20 \\
3\end{array}$ & II & & 30 & $2 h$ & II & $\begin{array}{l}4041.10 \\
4848.45\end{array}$ & 2 & $20 h$ & III \\
\hline $\begin{array}{l}4325.28 \\
4326.40\end{array}$ & $5 h$ & 3 & $\begin{array}{r}\text { II } \\
\text { I }\end{array}$ & $\begin{array}{l}4589.22 \\
4590.84\end{array}$ & $\begin{array}{l}20 \\
30\end{array}$ & $?$ & $\begin{array}{l}1 \\
\text { I }\end{array}$ & $\begin{array}{l}\begin{array}{r}8488.40 \\
4851.17\end{array}\end{array}$ & 8 & 10 & II \\
\hline & & & & & & & & 4853. & 3 & & I \\
\hline $\begin{array}{l}4329.72 \\
\end{array}$ & 1 & & I & 4593. & 2 & & 1 & & & & \\
\hline $\begin{array}{l}\mathbf{4 3 3 6 . 4 8} \\
4339.10\end{array}$ & 2 & ${ }_{10}^{3 h ?}$ & II & $\begin{array}{l}4597.25 \\
4508.37\end{array}$ & 10 & $\begin{array}{r}2 \\
50\end{array}$ & II & 4871.64 & & $3 h$ & II \\
\hline 4344.21 & 2 & & I & 4610.22 & $2 h$ & & I & 4893.48 & 2 & & I \\
\hline 4344.2 & & $2 ?$ & II & 4610.66 & & $4 ?$ & II & $\begin{array}{l}4894.63 \\
4894.98\end{array}$ & $\begin{array}{r}50 \\
3\end{array}$ & 10 & II \\
\hline 4352.94 & $6 h$ & & I & 4618.53 & & $3 ?$ & II & & & & \\
\hline & 3 & & I & & $4 h$ & & 1 & $\begin{array}{l}4897.94 \\
403\end{array}$ & ......... & ${ }_{8 h}^{2}$ & II \\
\hline 4363.3 & 5 & 8 & II & $\begin{array}{l}4633.18 \\
4634\end{array}$ & 2 & $-15 h$ & 1 & $\begin{array}{l}4903.72 \\
4912.38\end{array}$ & 20 & & I \\
\hline $\begin{array}{r}4370.81 \\
\mathbf{4 3 7 1 . 4 2}\end{array}$ & $\begin{array}{l}5 \\
1 ?\end{array}$ & & $\begin{array}{r}\text { II } \\
\text { I }\end{array}$ & $\begin{array}{l}4634.04 \\
4641.62\end{array}$ & $\begin{array}{l}2 \\
2\end{array}$ & $15 h$ & $\begin{array}{l}\text { II } \\
\text { I }\end{array}$ & 4917.04 & & $4 h$ & II \\
\hline 4372.60 & & 1 & II & 4644.54 & 10 & 1 & & & & & \\
\hline & 4 & & I & & 10 & 1 & 1 & 4918.46 & 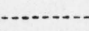 & $3 h ?$ & II \\
\hline & & $1 h$ & II & 465 & 3 & ...... & I & & & & II \\
\hline & & $2 h ?$ & II & & $9 h$ & & I & 4935.51 & 500 & 20 & I \\
\hline 4389.76 & 1 & 10 & II & 4658.47 & $1 ?$ & & I & 4936.96 & $8 h$ & & I \\
\hline 4392.83 & 2 & 15 & II & & $2 h$ & & I & 4937.22 & 5 & 100 & II \\
\hline & $30 h$ & & II & & 2 & $--\frac{-1}{7}$ & I & & $1 ?$ & & 1 \\
\hline & 1 & 2 & I & & 3 & 7 & II & 4942.78 & 1 & $5 h$ & II \\
\hline $\begin{array}{l}4398.95 \\
4402.30\end{array}$ & $\begin{array}{l}5 \\
4\end{array}$ & $\begin{array}{r}1 \\
20\end{array}$ & II & $\begin{array}{l}4683.83 \\
4684.27\end{array}$ & $\begin{array}{l}8 \\
7\end{array}$ & $\begin{array}{r}40 \\
1\end{array}$ & II & $\begin{array}{l}4944.09 \\
4944.96\end{array}$ & 5 & $\begin{array}{c}20 \\
7 h\end{array}$ & $\begin{array}{l}\text { II } \\
\text { II }\end{array}$ \\
\hline 4409.3 & 6 & 10 & II & & 1 & $4 h$ & II & 4954.67 & & $4 h$ & II \\
\hline & $1 ?$ & & I & & 1 & 5 & II & & $2 h$ & & 1 \\
\hline & & & I & 4702.36 & 3 & & 1 & & 2 & $\cdots$ & I \\
\hline & $10 h$ & $\cdots$ & I & 4704.90 & 3 & & I & 4966.91 & 100 & 4 & I \\
\hline 4430.22 & 6 & 1 & I & 4712.82 & & $30 h$ & II & 4972.30 & & $3 h ?$ & II \\
\hline 4439.21 & 100 & 10 & I & 4718.66 & $20 h$ & & I & 4973.90 & & $2 h ?$ & II \\
\hline 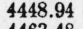 & $1 ?$ & & I & 4720.79 & 6 & 1 & I & 4974.16 & 10 & & I \\
\hline & & 2 & II & 4726.08 & 60 & 250 & II & 4988.30 & 4 & 1 & I \\
\hline $\begin{array}{l}4467.14 \\
4472.19\end{array}$ & & $\begin{array}{l}2 \\
3\end{array}$ & $\begin{array}{l}\text { II } \\
\text { II }\end{array}$ & $\begin{array}{l}4732.02 \\
4732.96\end{array}$ & 1 & $\begin{array}{l}7 \\
3\end{array}$ & $\begin{array}{l}\text { II } \\
\text { II }\end{array}$ & $\begin{array}{l}5009.53 \\
5014.50\end{array}$ & 20 & $\begin{array}{l}50 \\
10\end{array}$ & $\begin{array}{l}\text { II } \\
\text { II }\end{array}$ \\
\hline
\end{tabular}


TABLE 1.-Arc and spark spectra of ytterbium $(\mathrm{Z}=70)$ - Continued

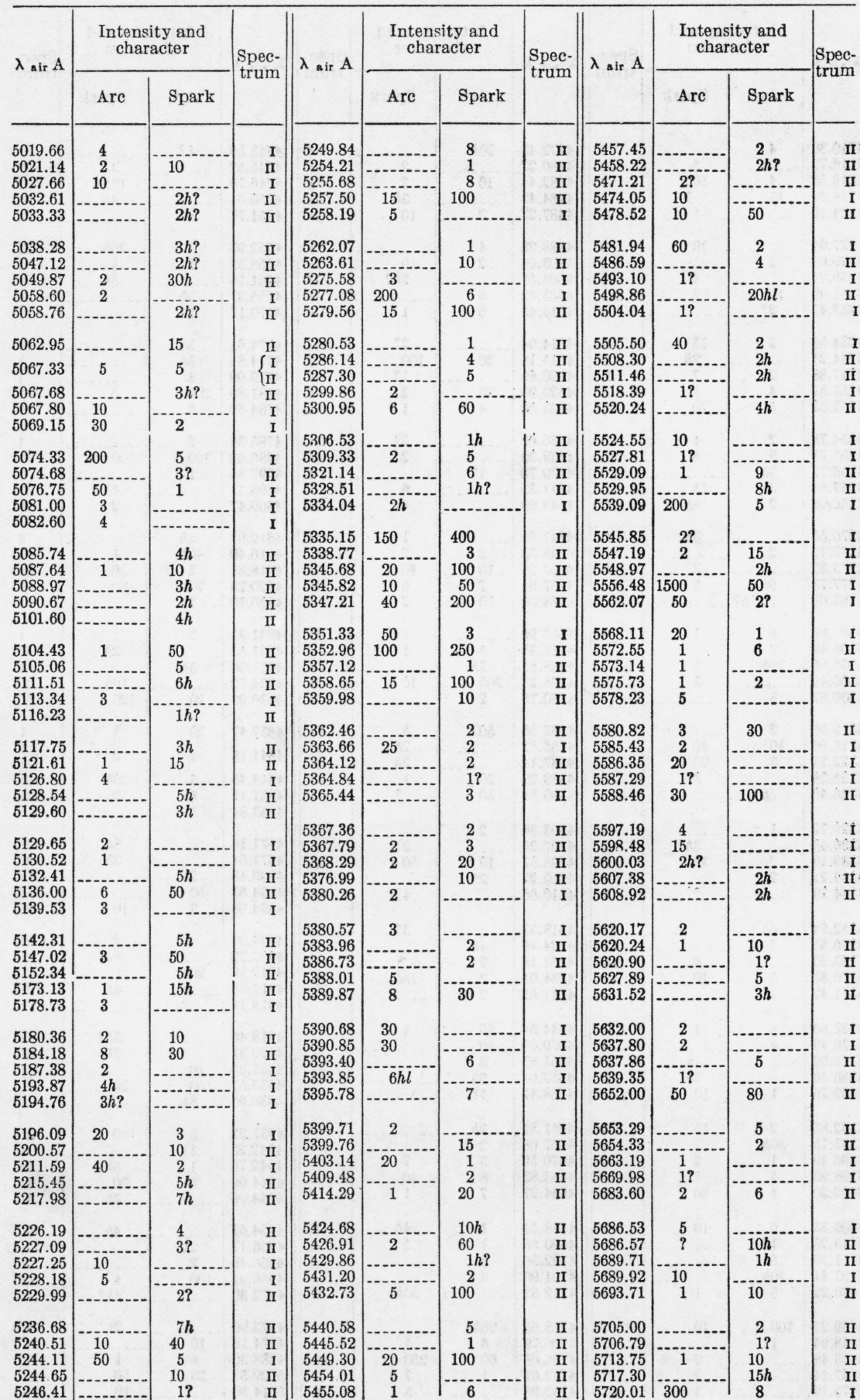


TABLE 1.-Arc and spark spectra of ytterbium $(\mathrm{Z}=70)$-Continued

\begin{tabular}{|c|c|c|c|c|c|c|c|c|c|c|c|}
\hline \multirow{2}{*}{$\lambda_{\text {air } \mathrm{A}}$} & \multicolumn{2}{|c|}{$\begin{array}{l}\text { Intensity and } \\
\text { character }\end{array}$} & \multirow{2}{*}{$\begin{array}{l}\text { Spec- } \\
\text { trum }\end{array}$} & \multirow{2}{*}{$\lambda$ ir $A$} & \multicolumn{2}{|c|}{$\begin{array}{l}\text { Intensity and } \\
\text { character }\end{array}$} & \multirow{2}{*}{$\begin{array}{l}\text { Spec- } \\
\text { trum }\end{array}$} & \multirow{2}{*}{$\lambda_{\text {a ir }} A$} & \multicolumn{2}{|c|}{$\begin{array}{l}\text { Intensity and } \\
\text { character }\end{array}$} & \multirow{2}{*}{$\begin{array}{l}\text { Spec- } \\
\text { trum }\end{array}$} \\
\hline & Arc & Spark & & & Arc & Spark & & & Arc & Spark & \\
\hline $\begin{array}{l}5723.72 \\
5728.86 \\
5730.02 \\
5735.80 \\
5736.95\end{array}$ & $\begin{array}{l}3 \\
5 \\
4 \\
2 \\
3 ?\end{array}$ & $\begin{array}{l}60 \\
15\end{array}$ & $\begin{array}{r}\text { I } \\
\text { I } \\
\text { II } \\
\text { II } \\
\text { I }\end{array}$ & $\begin{array}{l}6001.05 \\
6002.52 \\
6003.65 \\
6004.53 \\
6007.42\end{array}$ & $\begin{array}{l}5 \\
2 ? \\
4\end{array}$ & $\begin{array}{c}1 ? \\
2 ? \\
80 h\end{array}$ & $\begin{array}{r}\text { II } \\
\text { II } \\
\text { I } \\
\text { I } \\
\text { II }\end{array}$ & $\begin{array}{l}6312.99 \\
6324.42 \\
6324.73 \\
6324.78 \\
6333.36\end{array}$ & $\mid \begin{array}{c}-1 \\
3\end{array}$ & $\begin{array}{c}1 h ? \\
1 ? \\
2 \\
1 ?\end{array}$ & $\begin{array}{r}\text { II } \\
\text { II } \\
\text { II } \\
\text { I } \\
\text { II }\end{array}$ \\
\hline $\begin{array}{l}\mathbf{5 7 4 5 . 8 0} \\
\mathbf{5 7 4 9 . 9 2} \\
\mathbf{5 7 5 4 . 7 9} \\
\mathbf{5 7 5 5 . 9 0} \\
\mathbf{5 7 6 4 . 6 5}\end{array}$ & $\begin{array}{c}6 \\
8 \\
15 \\
-0\end{array}$ & $\begin{array}{c}10 \\
1 h ? \\
1 h ?\end{array}$ & $\begin{array}{r}\text { I } \\
\text { II } \\
\text { II } \\
\text { I } \\
\text { II }\end{array}$ & $\begin{array}{l}6014.95 \\
6020.56 \\
6021.97 \\
6024.08 \\
6031.80\end{array}$ & $\begin{array}{c}3 \\
2 \\
-2 \\
10\end{array}$ & $\begin{array}{c}5 \\
15 h \\
5 \\
-\end{array}$ & $\begin{array}{r}\text { I } \\
\text { II } \\
\text { II } \\
\text { II } \\
\text { I }\end{array}$ & $\begin{array}{l}6335.72 \\
6345.02 \\
6345.74 \\
6355.40 \\
6356.68\end{array}$ & $\begin{array}{c}8 \\
15 h \\
2 \\
2 \\
-\end{array}$ & $\begin{array}{c}7 \\
50 h \\
2 h ?\end{array}$ & $\begin{array}{l}\text { I } \\
\text { I } \\
\text { II } \\
\text { II } \\
\text { II }\end{array}$ \\
\hline $\begin{array}{l}5767.23 \\
5771.67 \\
5773.86 \\
5774.35 \\
5775.68\end{array}$ & $\begin{array}{r}2 \\
30 \\
2 \\
2 \\
\end{array}$ & \begin{tabular}{c}
$10 h$ \\
50 \\
\hdashline $1 ?$
\end{tabular} & $\begin{array}{r}\text { II } \\
\text { II } \\
\text { I } \\
\text { I } \\
\text { II }\end{array}$ & $\begin{array}{l}6035.72 \\
6040.80 \\
6042.26 \\
6048.43 \\
6052.88\end{array}$ & \begin{tabular}{c}
2 \\
3 \\
\hdashline 15 \\
8
\end{tabular} & $\begin{array}{c}10 \\
2 ? \\
30\end{array}$ & $\begin{array}{r}\text { I } \\
\text { II } \\
\text { II } \\
\text { I } \\
\text { II }\end{array}$ & $\begin{array}{l}6372.71 \\
6377.04 \\
6382.93 \\
6387.72 \\
6393.79\end{array}$ & $\begin{array}{c}6 \\
1 \\
3 \\
2 ?\end{array}$ & $\begin{array}{c}6 h \\
2 \\
\cdots\end{array}$ & $\begin{array}{r}\text { I } \\
\text { II } \\
\text { II } \\
\text { I } \\
\text { I }\end{array}$ \\
\hline $\begin{array}{l}\mathbf{5 7 7 8 . 0 0} \\
\mathbf{5 7 8 6 . 6 0} \\
\mathbf{5 7 8 9 . 9 4} \\
\mathbf{5 7 9 9 . 5 9} \\
\mathbf{5} 803.45\end{array}$ & $\begin{array}{l}5 \\
15\end{array}$ & $\begin{array}{c}3 \\
6 \\
2 h ? \\
\end{array}$ & $\begin{array}{r}\text { II } \\
\text { II } \\
\text { I } \\
\text { II } \\
\text { I }\end{array}$ & $\begin{array}{l}6054.56 \\
6056.48 \\
6059.25 \\
6075.25 \\
6082.40\end{array}$ & $\begin{array}{r}10 \\
1 \\
10 \\
\end{array}$ & $\begin{array}{c}20 h \\
3 \\
10 h\end{array}$ & $\begin{array}{r}\text { I } \\
\text { II } \\
\text { I } \\
\text { II } \\
\text { II }\end{array}$ & $\begin{array}{l}6400.40 \\
6402.64 \\
6404.62 \\
6417.97 \\
6421.02\end{array}$ & $\begin{array}{c}200 h \\
2 h \\
120 \\
1 ?\end{array}$ & \begin{tabular}{c}
$4 h$ \\
$1 h ?$ \\
\hdashline 3
\end{tabular} & $\begin{array}{r}\text { I } \\
\text { II } \\
\text { I } \\
\text { I } \\
\text { I }\end{array}$ \\
\hline $\begin{array}{l}5808.69 \\
5810.68 \\
5811.95 \\
5819.43 \\
5824.35\end{array}$ & 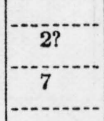 & $\begin{array}{c}2 \\
1 ? \\
100 \\
2 ?\end{array}$ & $\begin{array}{r}\text { II } \\
\text { I } \\
\text { II } \\
\text { II } \\
\text { II }\end{array}$ & $\begin{array}{l}6083.29 \\
6083.85 \\
6088.72 \\
6098.51 \\
6106.22\end{array}$ & 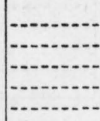 & $\begin{array}{l}2 \\
1 ? \\
3 \\
1 \\
4\end{array}$ & $\begin{array}{l}\text { II } \\
\text { II } \\
\text { II } \\
\text { II } \\
\text { II }\end{array}$ & $\begin{array}{l}6421.54 \\
6432.73 \\
6438.01 \\
6440.81 \\
6456.92\end{array}$ & $\begin{array}{r}3 \\
30 \\
- \\
- \\
-\end{array}$ & $\begin{array}{c}40 \\
1 h ? \\
5 \\
4\end{array}$ & $\begin{array}{l}\text { I } \\
\text { II } \\
\text { II } \\
\text { II } \\
\text { II }\end{array}$ \\
\hline $\begin{array}{l}5831.82 \\
5834.00 \\
5834.58 \\
5835.54 \\
5836.22\end{array}$ & $\begin{array}{c}1 \\
60 h \\
1 ?\end{array}$ & $\begin{array}{c}1 \\
1 ? \\
2 ?\end{array}$ & $\begin{array}{r}\text { I } \\
\text { I } \\
\text { I } \\
\text { II } \\
\text { II }\end{array}$ & $\begin{array}{l}6110.19 \\
6111.28 \\
6118.40 \\
6120.38 \\
6122.99\end{array}$ & $\frac{7}{10 h ?}$ & $\begin{array}{c}4 \\
-20 h \\
-\end{array}$ & $\begin{array}{r}\text { II } \\
\text { I } \\
\text { I } \\
\text { II } \\
\text { I }\end{array}$ & $\begin{array}{l}6463.15 \\
6474.74 \\
6488.50 \\
6489.10 \\
6489.27\end{array}$ & $\begin{array}{r}10 \\
5 \\
800^{?}\end{array}$ & $\begin{array}{r}100 \\
50 \\
1 \\
40 \\
30\end{array}$ & $\begin{array}{r}\text { II } \\
\text { II } \\
\text { II } \\
\text { I } \\
\text { II? }\end{array}$ \\
\hline $\begin{array}{l}5837.15 \\
5838.67 \\
5842.45 \\
5844.46 \\
5847.24\end{array}$ & $\begin{array}{c}50 \\
1 ? \\
2 ? \\
\end{array}$ & \begin{tabular}{c}
150 \\
2 \\
\hdashline $2 h$
\end{tabular} & $\begin{array}{r}\text { II } \\
\text { II } \\
\text { I } \\
\text { I } \\
\text { II }\end{array}$ & $\begin{array}{l}6123.03 \\
6126.37 \\
6128.21 \\
6131.61 \\
6134.31\end{array}$ & 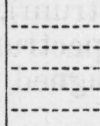 & $\begin{array}{l}4 \\
2 ? \\
6 \\
2 h ? \\
5\end{array}$ & $\begin{array}{l}\text { II } \\
\text { II } \\
\text { II } \\
\text { II } \\
\text { II }\end{array}$ & $\begin{array}{l}6492.74 \\
6503.02 \\
6509.11 \\
6523.24 \\
6541.37\end{array}$ & \begin{tabular}{c}
3 \\
2 \\
$2 ?$ \\
\hdashline $1 ?$
\end{tabular} & $\begin{array}{r}50 \\
4 \\
-4 ? \\
-\end{array}$ & $\begin{array}{r}\text { II } \\
\text { II } \\
\text { I } \\
\text { II } \\
\text { I }\end{array}$ \\
\hline $\begin{array}{l}\mathbf{5 8 5 4 . 5 2} \\
5865.65 \\
\mathbf{5} 868.40 \\
\mathbf{5} 874.70 \\
\mathbf{5 8 7 5 . 9 6}\end{array}$ & $\begin{array}{c}30 \\
1 \\
2 ?\end{array}$ & $\begin{array}{c}1 \\
2 \\
6 \\
30 h \\
-\end{array}$ & $\begin{array}{r}\text { I } \\
\text { II } \\
\text { II } \\
\text { II } \\
\text { I }\end{array}$ & $\begin{array}{l}6142.85 \\
6146.94 \\
6150.64 \\
6152.58 \\
6171.65\end{array}$ & $\begin{array}{l}30 h \\
60\end{array}$ & $\begin{array}{c}1 ? \\
15 h \\
-80 \\
2\end{array}$ & $\begin{array}{r}\text { II } \\
\text { II } \\
\text { I } \\
\text { II } \\
\text { II }\end{array}$ & $\begin{array}{l}6550.19 \\
6553.34 \\
6555.15 \\
6568.35 \\
6585.42\end{array}$ & $\begin{array}{r}10 \\
2 \\
3 \\
2 \\
7\end{array}$ & $\begin{array}{r}? \\
? \\
? \\
?\end{array}$ & $\begin{array}{r}\text { I } \\
\text { I } \\
\text { I } \\
\text { I } \\
\text { II }\end{array}$ \\
\hline $\begin{array}{l}\mathbf{5} 882.81 \\
\mathbf{5} 896.61 \\
\mathbf{5} 897.22 \\
\mathbf{5} 898.80 \\
\mathbf{5} 903.37\end{array}$ & $\begin{array}{l}2 \\
5 \\
7 \\
3\end{array}$ & $\begin{array}{c}10 \\
100 h \\
50 h \\
2\end{array}$ & $\begin{array}{r}\text { II } \\
\text { I } \\
\text { II } \\
\text { II } \\
\text { II }\end{array}$ & $\begin{array}{l}6175.59 \\
6189.07 \\
6190.81 \\
6194.85 \\
6208.11\end{array}$ & $\begin{array}{r} \\
3 \\
3 \\
2\end{array}$ & $\begin{array}{c}1 \\
1 ? \\
15 \\
20 h\end{array}$ & $\begin{array}{r}\text { II } \\
\text { II } \\
\text { II } \\
\text { I } \\
\text { II }\end{array}$ & $\begin{array}{l}6592.75 \\
6605.93 \\
6607.07 \\
6617.06 \\
6643.54\end{array}$ & $\begin{array}{l}20 \\
10 \\
50\end{array}$ & $\begin{array}{c}3 ? \\
4 h \\
-6\end{array}$ & $\begin{array}{r}\text { II } \\
\text { II } \\
\text { I } \\
\text { I } \\
\text { I }\end{array}$ \\
\hline $\begin{array}{l}5908.36 \\
5920.39 \\
5927.70 \\
5935.06 \\
5936.47\end{array}$ & $\begin{array}{r}20 \\
1 \\
2 \\
3 \\
2\end{array}$ & $\begin{array}{c}30 \\
10 \\
40 \\
-\end{array}$ & $\begin{array}{r}\text { II } \\
\text { II } \\
\text { I } \\
\text { II } \\
\text { I }\end{array}$ & $\begin{array}{l}6208.68 \\
6215.57 \\
6223.65 \\
6233.38 \\
6234.86\end{array}$ & & $\begin{array}{c}1 ? \\
6 \\
15 \\
5 \\
5\end{array}$ & $\begin{array}{l}\text { II } \\
\text { II } \\
\text { II } \\
\text { II } \\
\text { II }\end{array}$ & $\begin{array}{l}6644.07 \\
6661.90 \\
6667.85 \\
6678.17 \\
6679.32\end{array}$ & \begin{tabular}{c}
2 \\
1000 \\
20 \\
\hdashline
\end{tabular} & $\begin{array}{c}5 h \\
4 h ? \\
20 \\
4 ?\end{array}$ & $\begin{array}{r}\text { II } \\
\text { II } \\
\text { I } \\
\text { I } \\
\text { II }\end{array}$ \\
\hline $\begin{array}{l}5943.55 \\
5946.02 \\
5947.28 \\
5950.65 \\
5950.98\end{array}$ & \begin{tabular}{c}
1 \\
4 \\
1 \\
3 \\
\hdashline
\end{tabular} & $\begin{array}{r}2 \\
100 \\
5 \\
\hdashline 8\end{array}$ & $\begin{array}{r}\text { II } \\
\text { II } \\
\text { II } \\
\text { I } \\
\text { II }\end{array}$ & $\begin{array}{l}6235.27 \\
6236.15 \\
6236.56 \\
6246.97 \\
6247.99\end{array}$ & $\begin{array}{r}2 \\
4 \\
40 \\
3\end{array}$ & 6 & $\begin{array}{r}\text { I } \\
\text { II } \\
\text { I } \\
\text { II } \\
\text { I }\end{array}$ & $\begin{array}{l}6692.42 \\
6699.38 \\
6715.79 \\
6727.62 \\
6745.22\end{array}$ & $\begin{array}{c}2 ? \\
10 \\
5 \\
30 \\
2\end{array}$ & $\begin{array}{r}8 \\
-60 \\
77\end{array}$ & $\left\{\begin{array}{r}\text { I } \\
\text { I } \\
\text { II? } \\
\text { I } \\
\text { II } \\
\text { II }\end{array}\right.$ \\
\hline $\begin{array}{l}\mathbf{5} 955.35 \\
\mathbf{5 9 5 8 . 7 0} \\
\mathbf{5 9 5 9 . 3 1} \\
\mathbf{5} 961.85 \\
\mathbf{5 9 7 2 . 7 1}\end{array}$ & $\begin{array}{r}2 \\
10 \\
3 \\
1 ?\end{array}$ & $2 h ?^{-1}$ & $\begin{array}{r}\text { I } \\
\text { I } \\
\text { I } \\
\text { II } \\
\text { I }\end{array}$ & $\begin{array}{l}6260.80 \\
6265.46 \\
6270.33 \\
6271.16 \\
6274.79\end{array}$ & $\begin{array}{c}4 \\
100\end{array}$ & $\begin{array}{r}50 h \\
1 ? \\
1 ? \\
3 \\
150\end{array}$ & $\begin{array}{l}\text { II } \\
\text { II } \\
\text { II } \\
\text { II } \\
\text { II }\end{array}$ & $\begin{array}{l}6749.40 \\
6755.42 \\
6768.70 \\
6777.22 \\
6782.17\end{array}$ & $\begin{array}{c}7 \\
80 \\
2 \\
4 h\end{array}$ & $\begin{array}{c}4 ?^{2} \\
2 \\
-\end{array}$ & $\begin{array}{r}\text { I } \\
\text { II } \\
\text { I } \\
\text { I } \\
\text { I }\end{array}$ \\
\hline $\begin{array}{l}5985.35 \\
5986.80 \\
5987.91 \\
5989.32 \\
5991.51\end{array}$ & $\begin{array}{c}1 \\
-2 \\
15 \\
50\end{array}$ & -\begin{tabular}{c}
5 \\
$2 h ?$ \\
$20 h$ \\
\hdashline 150
\end{tabular} & $\begin{array}{r}\text { II } \\
\text { II } \\
\text { II } \\
\text { I } \\
\text { II }\end{array}$ & $\begin{array}{l}6277.13 \\
6286.26 \\
6297.38 \\
6303.28 \\
6308.16\end{array}$ & \begin{tabular}{|c|}
8 \\
3 \\
20
\end{tabular} & $\begin{array}{c}5 \\
15 h \\
4 \\
30\end{array}$ & $\begin{array}{l}\text { II } \\
\text { I } \\
\text { II } \\
\text { II } \\
\text { II }\end{array}$ & $\begin{array}{l}6785.16 \\
6790.82 \\
6799.61 \\
6802.47 \\
6813.66\end{array}$ & $\begin{array}{c}1 \\
1 ? \\
1000 \\
2 \\
1 ?\end{array}$ & $\begin{array}{c}5 \\
50 \\
20 \\
-0 .\end{array}$ & $\begin{array}{r}\text { II } \\
\text { I } \\
\text { I } \\
\text { II } \\
\text { I }\end{array}$ \\
\hline
\end{tabular}


TABLE 1.-Arc and spark spectra of ytterbium $(\mathrm{Z}=70)$-Continued

\begin{tabular}{|c|c|c|c|c|c|c|c|c|c|c|c|}
\hline \multirow{2}{*}{$\lambda$ air $\mathbf{A}$} & \multicolumn{2}{|c|}{$\begin{array}{l}\text { Intensity and } \\
\text { character }\end{array}$} & \multirow{2}{*}{$\begin{array}{l}\text { Spec- } \\
\text { trum }\end{array}$} & \multirow{2}{*}{$\lambda_{\text {a ir }} \mathbf{A}$} & \multicolumn{2}{|c|}{$\begin{array}{l}\text { Intensity and } \\
\text { character }\end{array}$} & \multirow{2}{*}{$\begin{array}{l}\text { Spec- } \\
\text { trum }\end{array}$} & \multirow{2}{*}{$\lambda=\operatorname{sir} \Lambda$} & \multicolumn{2}{|c|}{$\begin{array}{l}\text { Intensity and } \\
\text { character }\end{array}$} & \multirow{2}{*}{$\begin{array}{l}\text { Spec- } \\
\text { trum }\end{array}$} \\
\hline & Arc & Spark & & & Arc & Spark & & & Arc & Spark & \\
\hline 6820.04 & $3 h$ & & I & 7175.14 & 10 & & & 8053.41 & 10 & & \\
\hline 6826.06 & & $2 ?$ & II & 7187.06 & 5 & & & 8607.51 & 8 & & \\
\hline $\begin{array}{l}6828.90 \\
6833.57\end{array}$ & 1 & 6 & II & 7222.72 & 3 & & $\ldots$ & 8922.61 & 20 & $-\cdots$ & I \\
\hline 6864.26 & & $10 h ?$ & II & 7244.47 & 20 & & -.... & 9104.06 & 3 & & \\
\hline 6871.54 & 10 & & I & $\begin{array}{l}7250.8 \\
7305.25\end{array}$ & 15 & $\cdots$ & $\ldots$ & 9304.44 & 15 & & \\
\hline 6877.94 & & $8 h ?$ & II & 7313.10 & 20 & & $\ldots$ & 9349.27 & 20 & & $\cdots$ \\
\hline $\begin{array}{l}6878.12 \\
6881.43\end{array}$ & $3 ?$ & $4 h$ ? & I & 7342.3 & $2 ?$ & & $\cdots$ & 9392.8 & $\begin{array}{l}5 \\
4\end{array}$ & $\cdots$ & $\cdots$ \\
\hline 6889.60 & & $4 ?$ & II & 7350.09 & 40 & & & 9677.8 & $2 ?$ & & \\
\hline 6913.55 & $2 ?$ & & I & $\begin{array}{l}7361.8 \\
7377.55\end{array}$ & $\begin{array}{l}1 ? \\
4\end{array}$ & & 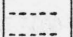 & 9688.6 & $3 ?$ & & \\
\hline 6934.04 & 15 & 10 & I, & 7405.92 & 3 & & 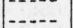 & 9760.37 & 100 & & \\
\hline 6951.51 & 2 & 10 & II? & 7422.1 & $4 ?$ & & -.... & 9799.88 & 10 & -... & $\ldots$ \\
\hline $\begin{array}{l}0901.01 \\
6963.11\end{array}$ & $\begin{array}{l}2 \\
2\end{array}$ & 5 & I & 7448.33 & 30 & & & $\begin{array}{l}9870.07 \\
0906640\end{array}$ & 6 & $\cdots$ & \\
\hline 6977.68 & 2 & & I & 7527.56 & 80 & & I & & & & \\
\hline & & & & 7699.49 & 1500 & & I & 10267.36 & & $-\cdots$ & $\cdots$ \\
\hline 6982.00 & 3 & 2 & $\mathrm{I}, \mathrm{II} ?$ & 7722.58 & 2 & $\mid \cdots$ & $\cdots$ & 10321.64 & 5 & -...... & \\
\hline $\begin{array}{l}6999.87 \\
7020.16\end{array}$ & $\begin{array}{r}15 \\
5\end{array}$ & 3 & & 7734.5 & $1 ?$ & & $-\ldots$ & 10770.1 & 2Lu? & & $-\cdots$ \\
\hline 7027.78 & $2 ?$ & $\cdots$ & 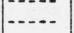 & 7758.03 & 10 & & -.... & & & & \\
\hline 7032.04 & 3 & & & $\begin{array}{l}7781.5 \\
7877.30\end{array}$ & $\begin{array}{l}1 ? \\
15\end{array}$ & & -...- & & & & \\
\hline 7032.91 & 2 & & -...-. & 7895.12 & 20 & $\ldots$ & 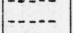 & & & & \\
\hline 7043.79 & 8 & & -..... & 7922.40 & 7 & $-\ldots$. & ....... & & & & \\
\hline
\end{tabular}

The data of table 1 represent at least three different spectra, each characteristic of $\mathrm{Yb}$ in some form or another. About 400 lines belong to neutral $\mathrm{Yb}$ atoms (Yb I spectrum), approximately 1,250 characterize singly ionized atoms (Yb II spectrum), and possibly a dozen belong to doubly ionized atoms (Yb III spectrum). We found no evidence of band heads which could be assigned to a molecular spectrum of ytterbium compounds.

The successive atomic spectra are differentiated mainly on the basis of intensity comparisons of arc and spark spectra, but since the latter were not recorded beyond 7,000 A the lines of longer wave length from ionized atoms remain unobserved, or unrecognized if recorded in arc spectra. The Yb I spectrum is surprisingly simple, but may not be fully developed in the arc. It may possess more lines in the infrared but the available $\mathrm{Yb}$ material was too scarce and precious to permit long exposures required to photograph beyond 10,500 $\mathrm{A}$. The Yb II spectrum is probably even more complex than is apparent because in addition to being undetermined beyond 7,000 $\mathrm{A}$ some lines are probably overlooked on account of being masked by silver lines or by the air spectrum.

Our assignment of lines to successive spectra agrees almost perfectly with King's [13] separation of ionization stages based upon a study of the electric-furnace spectra of $\mathrm{Yb}$. This grouping of lines is further confirmed by the spectral-term analyses, insofar as they have been carried out.

Since no details concerning regularities in the spectra of ytterbium have heretofore been published, we present a preliminary report, as follows: Soon after making our first measurements and identifications of lines from neutral $\mathrm{Yb}$ atoms, in 1930, we found from repeated differences among wave numbers three energy levels with separations of 703.5 and $1718.4 \mathrm{~cm}^{-1}$. These were interpreted as $\left(f^{14} s p\right)^{3} \mathrm{P}_{0,1,2}$ by 
Prof. H. N. Russell, who promptly identified two singlet levels $\left(f^{14} s p\right)^{1} \mathrm{P}_{1}$ and $\left(f^{14} s^{2}\right)^{1} \mathrm{~S}_{0}$, which account for the two lines of outstanding intensity and easy excitation in the $\mathrm{Yb}$ I spectrum. These terms and their combinations are displayed in table 2 where the term symbols and relative values appear on the margin and the wave lengths (intensities) and wave numbers of the observed combinations at intersections of lines and columns. Only the terms which can be interpreted with considerable certainty are given now, the remainder being reserved until the analysis is completed and confirmed by observations of Zeeman effects, which, up to the present time, have not been produced for $\mathrm{Yb}$ spectra. It appears certain that the normal state of neutral $\mathrm{Yb}$ atoms is described by $\left(4 f^{14} 6 s^{2}\right)^{1} \mathrm{~S}_{0}$ and that the most intense line of the $\mathrm{Yb}$ I spectrum is $\left(4 f^{14} 6 s^{2}\right)^{1} \mathrm{~S}_{0}-\left(4 f^{14} 6 s 6 p\right)^{1} \mathrm{P}_{1}$ with wave length $3,987.99 \mathrm{~A}$. This spectrum closely resembles that of an alkaline earth. Indeed, the fact that Yb may be bivalent accounts for its separation by electrolytic reduction from other trivalent rare earths [14].

Proceeding in a similar manner with $\mathrm{Yb}$ II lines the authors found several score of levels combining to produce some hundreds of lines, but it was impossible to group the levels into terms and identify them. Two lines of extraordinary intensity featuring the Yb II spectrum were not included among our level combinations and Professor Russell suggested that they represent the transition $\left(f^{14} s\right)^{2} \mathrm{~S}_{0}-\left(f^{14} p\right)^{2} \mathrm{P}_{1,2}$. When the $f$ shell is completely filled $\left(f^{14}\right)$ it contributes nothing to the spectrum and a single-valence electron will produce an alkali-like spectrum with $(s)^{2} \mathrm{~S}_{0}$ describing the normal state. However, the relatively great complexity of the Yb II spectrum indicates that most of the excited states must arise from electron configurations of types $f^{13} s^{2}, f^{13} s d, f^{13} d^{2}, f^{13} s p, f^{13} s d$, which produce large families of spectral terms. This is probably the explanation of the numerous levels first discovered, but further attempts to interpret them and connect them with terms arising from configurations with $f^{14}$ electrons will be postponed until Zeeman effects are available.

TABLE 2.-Terms and combinations in the $\mathrm{Yb}$ I spectrum

\begin{tabular}{|c|c|c|c|c|c|}
\hline \multicolumn{2}{|c|}{$\begin{array}{l}\text { Term symbol } \\
\text { Value }\end{array}$} & \multirow[t]{2}{*}{$\begin{array}{c}\left(4 f^{14} 6 s 6 p\right){ }^{3} \mathrm{P}_{0}^{\circ} \\
17288.5\end{array}$} & \multirow[t]{2}{*}{$\begin{array}{c}\left(4 f^{14} 6 s 6 p\right)^{3} \mathrm{P}_{1}^{\circ} \\
17992.0\end{array}$} & \multirow[t]{2}{*}{$\begin{array}{c}\left(4 f^{14686 p}\right){ }^{3} \mathrm{P}_{2}^{\circ} \\
19710.4\end{array}$} & \multirow[t]{2}{*}{$\begin{array}{l}\left(1 f^{14} 6 s 6 p\right) 1 \mathrm{P}_{1}^{\circ} \\
25068.2\end{array}$} \\
\hline Term symbol & Value & & & & \\
\hline$\left(4 f^{14} 6 s^{2}\right)^{1} S_{0} \ldots$ & 0.0 & & $\begin{array}{l}5556.48(1500) \\
17992.0\end{array}$ & & $\begin{array}{l}3987.98(2000) \\
25068.2\end{array}$ \\
\hline$\left(4 f^{14} 6 s 7 s\right)^{3} \mathrm{~S}_{1-\ldots}$ & 32694.7 & 15406.2 & 14702.7 & $\begin{array}{l}7699.49(1500) \\
12984.3\end{array}$ & \\
\hline$\left(4 f^{14} 6 s 5 d\right)^{3} \mathrm{D}_{1}$ & 39808.7 & $\begin{array}{l}4439.21(100) \\
22520.2\end{array}$ & $\begin{array}{l}4582.36(50) \\
21816.7\end{array}$ & $\begin{array}{l}4974.16(10) \\
20098.3\end{array}$ & $\begin{array}{l}6782.17(4) \\
4740.5\end{array}$ \\
\hline$\left(4 f^{14} 6 s 5 d\right)^{3} \mathrm{D}_{2}$ & 39838.0 & & $\begin{array}{l}4.576 .21(200) \\
21846.0\end{array}$ & $\begin{array}{l}4966.91(100) \\
20127.6\end{array}$ & $\begin{array}{l}6768.70(80) \\
4769.8\end{array}$ \\
\hline$\left(4 f^{14} 6 s 5 d\right)^{3} \mathrm{D}_{3 \ldots}$ & 39966.1 & & & $\begin{array}{l}4935.51(500) \\
20255.7\end{array}$ & \\
\hline$\left(4 f^{14} 6 s 5 d\right)^{1} \mathrm{D}_{2}$ & 40061.5 & & $\begin{array}{l}4529.90(20) \\
22069.4\end{array}$ & $\begin{array}{l}\text { 4912. } 38(20) \\
20351.1\end{array}$ & $\begin{array}{l}6667.85(1000) \\
4993.2\end{array}$ \\
\hline$\left(4 f^{14} 6 s 8 s\right)^{3} \mathrm{~S}_{1--}$ & 41614.9 & $\begin{array}{l}4109.60(5) \\
24326.4\end{array}$ & $\begin{array}{l}4231.99(10) \\
23622.9\end{array}$ & $\begin{array}{l}4564.00(50) \\
21904.5\end{array}$ & \\
\hline$\left(4 f^{14} 6 s 6 d\right)^{1} \mathrm{D}_{2} \ldots$ & 44760.3 & & $\begin{array}{l}3734.70(6) \\
26768.3\end{array}$ & $\begin{array}{l}3990.89(40) \\
25050.0\end{array}$ & $\begin{array}{l}5076.75(50) \\
19692.2\end{array}$ \\
\hline
\end{tabular}


The resonance lines of $\mathrm{Yb}$ II are interpreted with considerable certainty, as shown in table 3 . The most intense line emitted by singly ionized $\mathrm{Yb}$ atoms is expected to be $\left(f^{14} 6 s\right)^{2} \mathrm{~S}_{1 / 2}-\left(f^{14} 6 p\right)^{2} \mathrm{P}_{1 / 2}{ }^{1}$ with wave length $3289.36 \mathrm{~A}$, but the estimated intensities appear to be somewhat anomalous. Apparently $3694.19 \mathrm{~A}$ is the strongest $\mathrm{Yb}$ II line.

No attempt has been made to analyze the $\mathrm{Yb}$ III spectrum, but it may be expected that the ground state of doubly ionized $\mathrm{Yb}$ atoms is described by $\left(4 f^{14}\right)^{1} \mathrm{~S}_{0}$. The full shell of $f$-type electrons is likely to have high stability on account of which the fundamental lines emitted by $\mathrm{Yb}^{++}$ions will probably lie in the extreme ultraviolet.

TABLE 3.-Terms and combinations in the $\mathrm{Yb}$ II spectrum

\begin{tabular}{|c|c|c|c|}
\hline \multicolumn{2}{|c|}{$\begin{array}{l}\text { Term symbol_..... } \\
\text { Value }\end{array}$} & \multirow[t]{2}{*}{$\begin{array}{l}\left(4 f^{14} 6 p\right)^{2} \mathrm{P}^{\circ} 0^{1 / 6} \\
27061.9\end{array}$} & \multirow[t]{2}{*}{$\begin{array}{l}\left(4 f^{146} p\right)^{2} \mathrm{P}^{\circ}{ }_{13 / 4} \\
30392.3\end{array}$} \\
\hline Term symbol & Value & & \\
\hline$\left(4 f^{1468}\right)^{2} \mathrm{~S}_{03 / 6}$ & 0.0 & $\begin{array}{l}3694.19(1000) \\
27061.9\end{array}$ & $\begin{array}{l}3289.36(800) \\
30392.3\end{array}$ \\
\hline$\left(4 f^{147} 78\right)^{2} \mathrm{~S}_{03 / 6}$ & 54304.3 & $\begin{array}{l}3669.71(10) \\
27242.4\end{array}$ & $\begin{array}{l}4180.82(100) \\
23912.0\end{array}$ \\
\hline
\end{tabular}

\section{REFERENCES}

[1] C. Marignac, Compt. rend. 87, 578 (1878).

[2] G. Urbain, Compt. rend. 145, 759 (1907).

[3] C. Auer v. Welsbach, Sitzber Akad. Wiss. Wien 116 IIb, 1425 (1907).

[4] W. F. Meggers and B. F. Scribner, BS J. Research 19, 31 (1937). RP 1008.

[5] For earlier history see: H. Kayser, Handbuch der Spectroscopie 6, 192 (S. Hirzel, Leipzig, 1912).

[6] J. M. Eder and E. Valenta, Sitzber. Akad. Wiss. Wien 119, IIa, 6 (1910).

[7] J. M. Eder and E. Valenta, Atlas typischer Spektren, Wien Akad. (1911).

[8] F. Exner and E. Haschek, Spektren der Elemente bei normalem Druck 2, 2 (Franz Deuticke, Wien, 1911).

[9] F. Exner and E. Haschek, Spektren der Elemente bei normalem Druck 3, 4 (Franz Deuticke, Wien, 1911).

[10] J. Blumenfeld and G. Urbain, Compt. rend. 159, 401 (1914).

[11] J. M. Eder, Sitzber. Akad. Wiss. Wien 124, IIa, 707 (1915).

[12] H. Kayser, Tabelle der Hauptlinien der Linienspektra aller Elemente (J. Springer, Berlin, 1925).

[13] A. S. King, Astrophys. J. 74, 328 (1931).

[14] R. W. Ball with L. F. Yntema, J. Am. Chem. Soc. 52, 4264 (1930).

D. W. Pierce, C. R. Naeser, with B. S. Hopkins, Trans. Electrochem. Soc. 69, 557 (1936).

[15] W. F. Meggers, BS J. Research 9, 239 (1932.) RP 468.

Washington, Sept. 29, 1937. 